\title{
Quatre espèces nouvelles de Diplopodes cavernicoles de l'Andalousie (Espagne) (Diplopoda: Polydesmida: Polydesmidae; Chordeumatida: Chamaesomatidae, Opisthocheiridae)
}

\author{
Four new species of cavernicolous millipedes from Andalusia, Spain \\ (Diplopoda: Polydesmida: Polydesmidae; Chordeumatida: \\ Chamaesomatidae, Opisthocheiridae)
}

\section{Четыре новых вида пещерных двупарноногих многоножек из Андалусии (Испания) (Diplopoda: Polydesmida: Polydesmidae; Chordeumatida: Chamaesomatidae, Opisthocheiridae)}

\author{
Jean-Paul Mauriès \\ Жан-Помь Морьес
}

Attaché honoraire au Muséum national d'Histoire naturelle, Dpt. Systématique et Evolution, section Arthropodes, C.P. n 53,61 rue Buffon, F-75231 Paris Cedex 05, France.

Национальный музей естественной истории, Париж, Франция.

KEY WORDS: Diplopoda, taxonomy, new species, caves, Spain.

MOTS-CLES: Diplopoda, taxonomie, espèces nouvelles, grottes, Espagne.

КЛЮЧЕВЫЕ СЛОВА: Diplopoda, таксономия, новый вид, пещера, Испания.

RÉSUMÉ. Descriptions de quatre nouvelles espèces de Diplopodes, identifiées parmi le matériel récolté principalement en Andalousie (Espagne) dans des cavités situées dans les Parc Naturels des provinces de Jaén et de Granada et dans la province voisine d'Albacete par le "Grupo de Espeleología de Villacarillo". Ces nouveaux taxa appartiennent à trois familles et à trois genres distincts: Archipolydesmus giennensis sp.n. est un Polydesmida, Polydesmidae. Les trois autres espèces sont des Chordeumatida: Origmatogona toniperezi sp.n. est un Chamaesomatidae, et les deux Ceratosphys, C. flammeola sp.n. et C.fernandoi sp.n. appartiennent à la famille des Opisthocheiridae. Une clé sommaire pour les espèces du genre $\mathrm{Ar}$ chipolydesmus et un supplément à une clé récemment parue pour les espèces du genre Ceratosphys figurent dans cette note. Le genre endémique andalou Beticosoma Mauriès, 1990 est exclu des Chamaesomatidae, Scutogoninae, Origmatogonini, et devient le monotype d'une nouvelle famille des Beticosomatidae.

ABSTRACT. Four new species of Diplopoda are identified and described from among material collected principally in Andalusia, Spain in caves located in natural parks of the Jaén and Granada provinces, as well in the adjacent Albacete province, by the "Grupo de Espeleología de Villacarillo". These new taxa are classified in three genera from three distinct families.
Archipolydesmus giennensis sp.n. belongs to the Polydesmidae, Polydesmida. The other three new species are all in the order Chordeumatida: Origmatogona toniperezi sp.n. is in Chamaesomatidae while both Ceratosphys, C. flammeola sp.n. and C. fernandoi sp.n., are in Opisthocheiridae. A key to species Archipolydes$m u s$ is given, coupled with a supplement to the key published recently by the author for the genus Ceratosphys. The endemic Andalusian genus Beticosoma Mauriès, 1990 is excluded from the Chamaesomatidae, Scutogoninae, Origmatogonini to becomes the type of a new monotypic family Beticosomatidae, fam.n.

РЕЗЮМЕ. В материале многоножек-диплопод, в основном собранном «Спелеологической группой Вильякарильо» в пещерах Андалусии (Испания), расположенных в национальных парках провинций Хаэн и Гранада, а также в соседней провинции Албасете, выявлены и описаны четыре новых вида. Эти новые таксоны принадлежат трем родам из трех разных семейств. Archipolydesmus giennensis sp.n. принадлежит Polydesmidae, Polydesmida. Прочие три вида из отряда Chordeumatida: Origmatogona toniperezi sp.n. из Chamaesomatidae, а оба вида Ceratosphys, C. flammeola sp.n. и C. fernandoi sp.n., из Opisthocheiridae. Дан ключ для видов species Archipolydesmus, а также даны дополнения к ключу для видов рода Ceratosphys. Эндемичный в Анда- 
лусии род Beticosoma Mauriès, 1990 исключен из Chamaesomatidae, Scutogoninae, Origmatogonini и стал типовым в составе нового монотипического семейства Beticosomatidae, fam.n.

\section{Introduction}

La présente note fait suite à deux notes récentes [Mauriès, 2013b; Golovatch \& Mauriès, 2013] consacrées à des Diplopodes récoltés, entre 1985 et 2002 , dans plusieurs cavités de l'Andalousie mais aussi de la province voisine d'Albacete par le très actif "Grupo de Espeleología de Villacarillo" (GEV), de la province de Jaén, dirigé et animé par Antonio Pérez Fernàndez. Ce dernier a effectué récemment un nouvel envoi de matériaux récoltés entre 2008 et 2013, en leg pour le Musée Zoologique de Moscou, en direction de notre ami et collègue Serge Golovatch, qui a bien voulu m'en confier l'étude. Ce matériel recèle très majoritairement des Chordeumida, un seul Polydesmida et quelques Julida; ces derniers ne sont pas identifiables en l'absence de mâles adultes. Quelques-unes des espèces identifiées de ce dernier leg sont déjà connues de la région (comme le montre le tableau en Annexe) et quatre sont nouvelles pour la Science. Ces dernières font l'objet de la présente note; elles appartiennent, l'une, Archipolydesmus giennensis sp.n., à la famille des Polydesmidae (ordre Polydesmida), et les trois autres, qui appartiennent à l'ordre des Chordeumatida, se trouvent dans deux familles distinctes: Origmatogona toniperezi sp.n. est un Chamaesomatidae, tandis que les deux Ceratosphys, C. flammeola sp.n. et C. fernandoi sp.n., sont des Opisthocheridae.

La presque totalité de ce matériel sera déposée au Musée Zoologique de Moscou, à l'exception de quelques exemplaires de Origmatogona toniperezi sp.n. et de Ceratosphys fernandoi sp.n. qui seront déposés au MNHN de Paris.

\section{Partie systématique \\ POLYDESMIDA}

\section{Polydesmidae}

\section{Le genre Archipolydesmus Attems, 1898}

Connu du Maroc (une espèce), d'Algérie (3 espèces), d'Espagne (5 espèces, dont une d'Andalousie) et de Catalogne française (une espèce), ce genre a fait l'objet récemment d'une succinte mise au point [Mauriès, 2013b], dans laquelle a été décrite $A$. cordubaensis, de la province de Córdoba, et où a été signalée une espèce non identifiée au niveau spécifique dans la province de Cádiz.

N.B. Il est bon de signaler ici que, dans son travail sur les Diplopodes de Catalogne, Vicente [1981] a figuré et identifié sous le nom de Archipolydesmus panteli des spécimens récoltés par elle-même dans les provinces de Lérida et Tarragona. Or, les figures pu- bliées ne montrent ni un nombre de tubercules tergaux, ni un aspect de télopodes gonopodiaux correspondant à la description de panteli, qui d'ailleurs se situe géographiquement bien plus vers le sud-ouest, en Castille. Une partie au moins des exemplaires cités par Vicente, ceux qu'elle a figuré, appartiennent très probablement à un taxon inédit, qui d'après le nombre de tubercules tergaux, se rapprocherait des autres taxa catalans (ribauti et osellai), mais qui s'en distinguerait par la structure des gonopodes. Ce matériel, qui est certainement déposé à Barcelone, mériterait d'être revu.

\section{Archipolydesmus giennensis sp.n. Figs 1-8.}

Loc. type: Espagne, Andalousie, prov. Jaén, Hornos, Sima del Campamento, 03-03-2013, coll. Grupo de Espeleología de Villacarillo, $O^{7}$ holotype.

Nom: dénomination latine de la région de Jaén.

\section{Caractères morphologiques externes}

20 anneaux. Pigmentation brunâtre-grisâtre uniforme, les pattes légèrement plus claires.

Formule porifère banale: ozopores très discrets (sauf sur le T.V) s'ouvrant dorsalement dans la $3^{\text {ème }}$ denticulation du bord latéral des paranota des anneaux V, VII, IX, X, XII, XIII, XV à XVIII. Le T.XIX est sans paranota et sans ozopore.

Quelques largeurs (en $\mathrm{mm}$ )

Tête: 1,31 ; collum: 1,$50 ; 2^{\text {ème }}$ anneau (T.II): 1,52; prozonite du T.III: 0,70; métazonite du T.III: 1,38; métazonite du T.IV: 1,65; métazonite du T.V: 1,73; métazonite du T.VI: 1,91; prozonite du T.VII: 1,38; métazonite du T.VII: 2,00; prozonite des T.VIII et suivants: 1,42; métazonite des T.VIII et suivants: 2,03.

Tête globuleuse, sans particularité notable, sans pilosité ni sillon occipital net. Seulement quelques très fines soies éparses sur les joues.

Antennes mesurant 2,37 mm de long; antennomères 2 à 6 faiblement claviformes, mesurant respectivement 0,15 / 0,34 / 0,60 / 0,42 / 0,47 mm, les deux terminaux ( $7 \& 8$ ) atteignant $0,56 \mathrm{~mm}$ de long.

Collum à arcature du bord oral presque parfaitement hémicirculaire et arcature du bord caudal faible. La marge orale est découpée en 9+9 lobules petits mais nets, apparemment non-sétifères: la surface est ornée de 4 rangs transverses (les 3 premiers arqués) de $4+4$, $4+4,3+3$ et $2+2$ tubercules; ces tubercules sont petits, en granules, sauf le plus interne du $4^{\mathrm{ème}}$ rang, qui est nettement plus grand.

Autres anneaux:

Si le collum est apparemment glabre, on peut observer une faible pilosité sur les anneaux suivants: des soies très courtes et très fines existent sur quelques uns des tubercules des premier et troisième rangs des anneaux de la moitié antérieure du corps, notamment des T.IV aux T.XI. Sur les anneaux de la moitié postérieure du corps, on observe davantage de tubercules porteurs d'une soie; ces dernières deviennent aussi plus visibles.

Seules les paranota des anneaux T.II, III \& IV sont légèrement relevées; sur la plupart des anneaux, leurs 

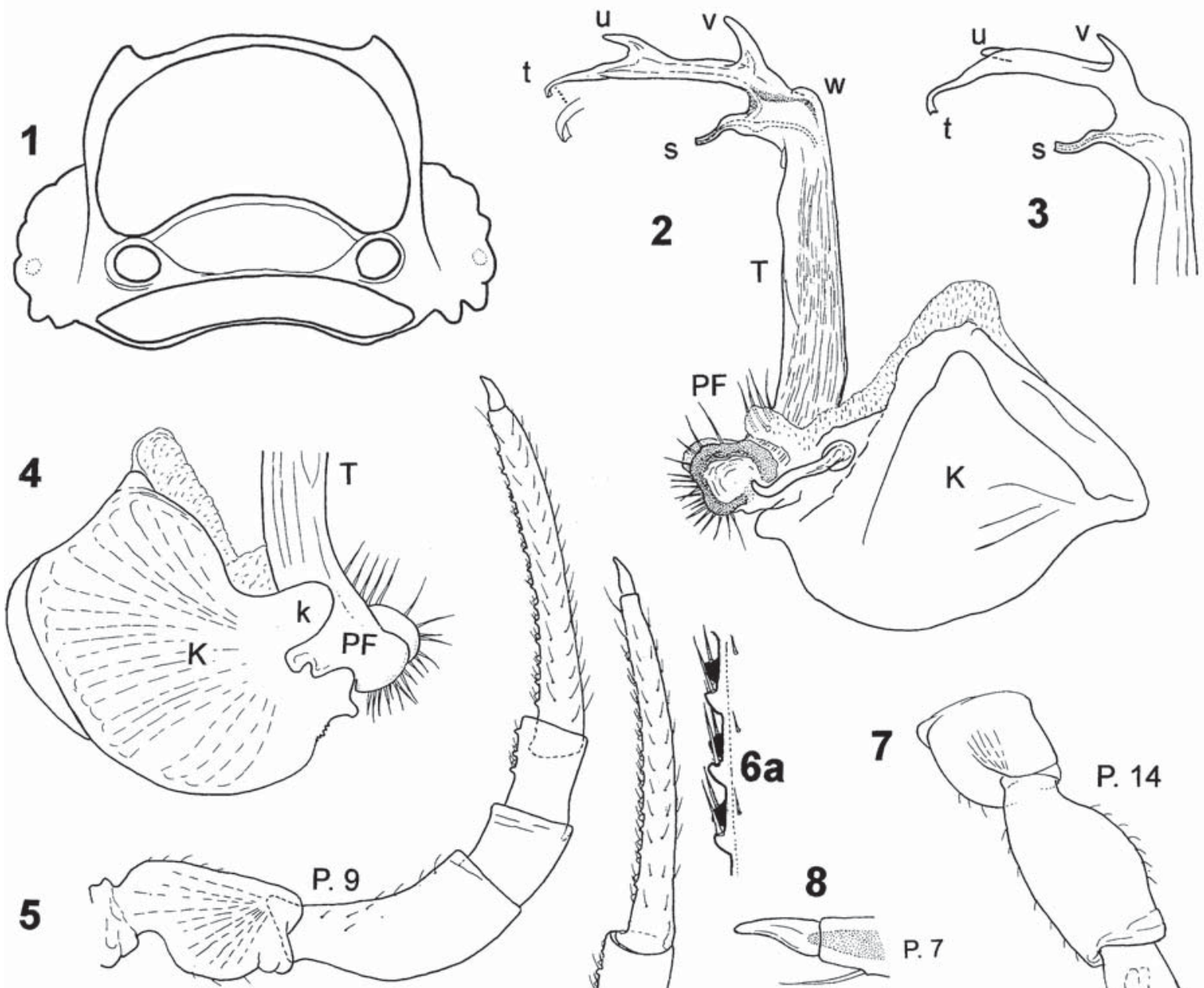

5
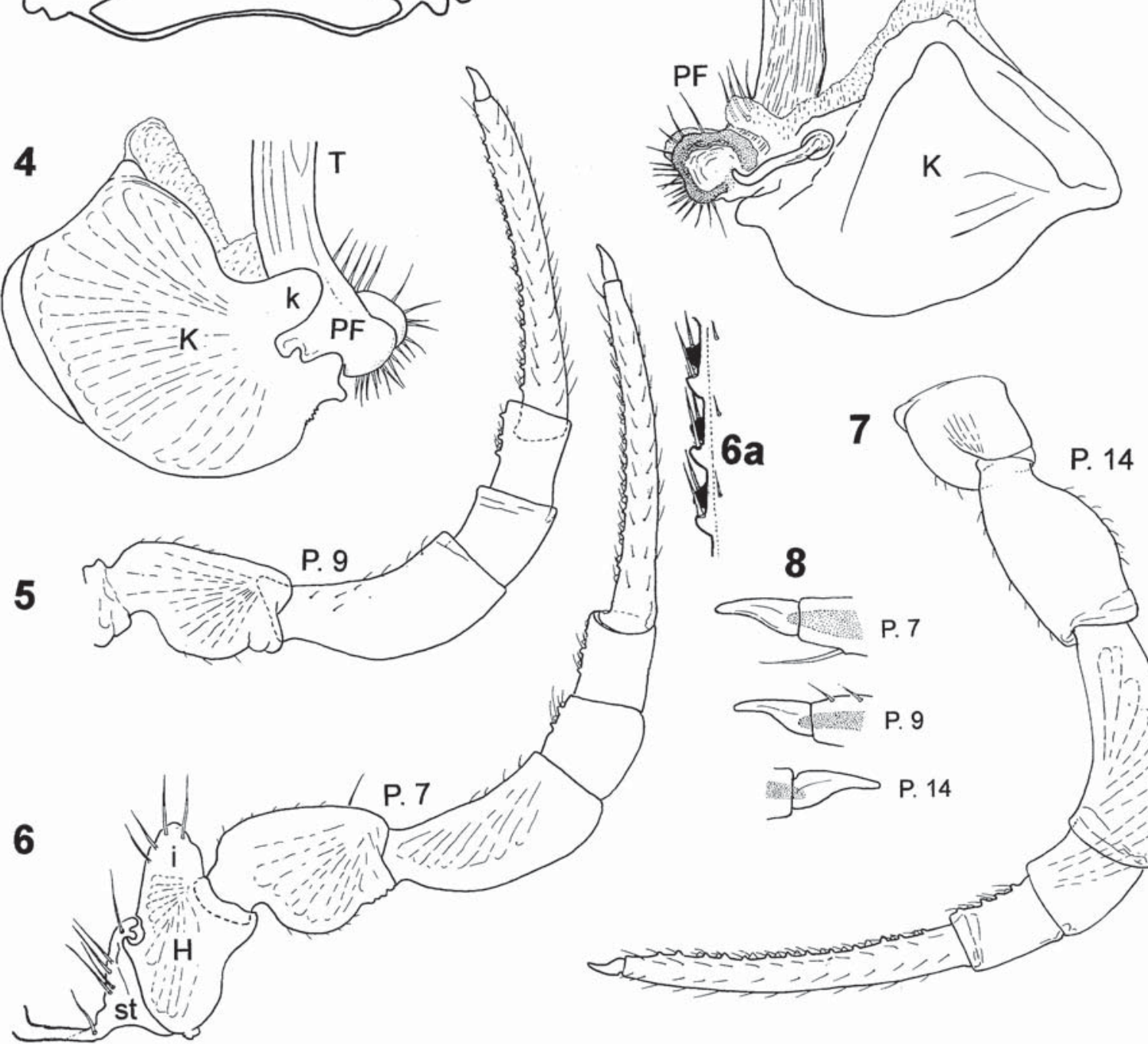

Fig. 1

Figs $2 \cdot 7$

Fig. 8

Figs 1-8. Archipolydesmus giennensis sp.n., holotype: 1 - vue ventrale, schématisée, du T.VII, gonopodes et P.9 extraits; 2 gonopode droit, vue médiale; 3 - autre aspect de la partie distale du même, vue latérale externe; 4 - hanche (face externe bombée), préfémur et base du télopodite du gonopode droit; 5 — P.9; 6 - P.7; 6a — fragment du métatarse de la P.7, grossi; 7 - P.14; 8 — uncus des P.7, 9 et 14. Echelles: 1,0 mm (1); 0,5 mm (2-7); 0,1 mm (8).

Figs 1-8. Archipolydesmus giennensis sp.n., holotype: 1 - ventral schematic view of tergite VII, gonopods and legs 9 extracted; 2 right gonopod, medial view; 3 - different aspect of distal part of the same, latero-external view; 4 - coxa (a convex external side), prefemur and base of right gonopod telopodite; $5-\operatorname{leg} 9 ; 6-\operatorname{leg} 7 ; 6 \mathrm{a}-$ fragment of metatarsus of leg 7, enlarged; 7 leg $14 ; 8-$ claw of legs 7, 9 and 14. Scale bars: $1.0 \mathrm{~mm}(1) ; 0.5 \mathrm{~mm}(2-7) ; 0.1 \mathrm{~mm}(8)$.

Рис. 1-8. Archipolydesmus giennensis sp.n., голотип: 1 - схематично снизу тергит 7, вычленены гоноподы и ноги 9; 2 правый гонопод, изнутри; 3 - иной вид его же дистальной части, сбоку снаружи; 4 - тазик (выпуклая наружная часть), пребедро и основание телоподита правого гонопода; 5 - нога $9 ; 6$ - нога 7; 6а - фрагмент метатарсуса 7, увеличено; 7 - нога $14 ; 8-$ коготок ног 7, 9 и 14. Масштаб: 1,0 мм (1); 0,5 мм (2-7); 0,1 мм (8). 
angles antérieurs et postérieurs ne présentent pas de forme angulaire en angle droit, sauf sur le T.II.

$\mathrm{Au}$ T.II, le bord latéral des paranota est divisé en 4 lobes sub-égaux (le postérieur un peu plus grand); le dos est parcouru transversalement par 3 rangs de petits tubercules granuliformes, $6+6$ au premier rang, 7+7 au $2^{2 \mathrm{ème}}$, et $4+4$ au marginal postérieur.

$\mathrm{Au}$ T.III, le bord oral des paranota est faiblement bi-denticulé et le bord latéral faiblement trilobé (le lobe postérieur un peu plus large). Le dos est parcouru par 3 rangs de tubercules: un premier rang de $6+6$ tubercules plats et allongés longitudinalement, un $2^{\text {ème }}$ de petits tubercules ronds, et un $3^{\text {ème }}$, marginal, de $5+5$ tubercules ronds.

Au T.IV, très semblable au précédent, le bord oral des paranota est lisse et il n'y a que $5+5$ grands tubercules allongés au premier rang.

Au T.V (premier porifère), le bord oral des paranota est faiblement bi-denticulé; le bord latéral est quadrilobé, les deux lobes antérieurs étant denticulés et les deux autres formant un bourrelet réniforme au milieu duquel s'ouvre l'ozopore. Des trois rangs transverses de tubercules, les deux premiers ont $5+5$ tubercules bas et allongés, tandis que le $3^{\text {ème }}$, marginal, constitue une frange de $5+5$ tubercules lobiformes.

Au T.VI, le bord oral des paranota n'est pas droit, mais forme un grand angle légèrement proéminent, et le bord latéral est quadridenticulé (dents courtes et émoussées). Le dos montre 3 rangs transverses de tubercules: $5+5$ tubercules bas et allongés sur les deux premiers rangs, $5+6$ (!) lobiformes sur le rang marginal postérieur.

A partir du T.VII ( $2^{\text {ème }}$ porifère), les paranota des autres porifères ont un bord oral lisse et le bord latéral est subdivisé en 4 courtes dents, la plus postérieure, où s'ouvre l'ozopore, étant un peu plus longue, mais non réniforme comme au T.V. A partir du T.VIII, et sur les autres non-porifères, les paranota ont aussi leur bord oral lisse et leur bord latéral quadri-denté.

Les rangs transverses de T.VII et T.VIII ont respectivement, du premier au $3^{\text {ème }}, 5+5,6+6$ et $6+6$ tubercules, mais, à partir du T.IX, le premier et le $3^{\text {ème }}$ rangs possèdent $6+6$ tubercules, et, au $2^{\text {ème }}$ rang, les tubercules tendent à s'effacer, et la moitié externe de cette zone, qui correspond à l'espace occupé normalement par 3 tubercules, est remplacée, de chaque côté, par une aire subcirculaire plus large, glabre, lisse et légèrement bombée.

Pygidium et valves anales sans particularité notable.

Uncus (Fig. 8) très simples.

\section{Caractères sexuels du mâle}

Pattes (des P.3 aux P.19) à tarses et métatarses pourvus d'une double rangée interne de granules (ou papilles) sétifères; on en compte $3+3$ ou $3+2$ aux tarses et $15+13$ aux métatarses (Figs 5-7). Aux P.7 (Fig. 6), il y en a même $3+2$ au tibia! Ces rangs de granules ont tendance à s'estomper puis disparaître dans le dernier tiers du corps.

N.B. De telles double-rangées de granules sétifères métatarsaux avaient été signalées, décrites et dessinées (dessin inédit qui se trouve dans le fichier iconographique du MNHN de Paris) par Brolemann [1926] dans la description du mâle de $A$. ribauti. Depuis cette date, seul Attems [1952] avait noté, dans la diagnose de son genre Miradoria, la présence de granules sétifères tarsaux chez les mâles; mais il semble n'en avoir vu et dessiné (sa fig. 30) qu'une seule rangée. La présence de ces doublerangées chez la nouvelle espèce permet de supposer qu'elles ont été non vues ou négligées par les auteurs et qu'en fait, elles existent chez les mâles de toutes les espèces du genre. C'est ce que l'auteur a pu vérifier sur 7 espèces: outre ribauti et giennensis, ces structures existent chez chreensis, cordubaensis, fodili, kabylianus et panteli. Les femelles en sont apparemment toutes dépourvues, mais l'auteur n'a pu le vérifier (outre ribauti et giennensis) que sur cinq autres espèces: cordubaensis, bedeli, chreeensis, fodoli, et panteli.

Au T.VI, les P.6 et P.7, qui sont (comme les P.3, 4 et 5), plus robustes que les autres paires de pattes, ont leur préfémur un peu renflé et surtout leurs hanches nettement écartées l'une de l'autre médialement (plus aux T.7 qu'aux T.6), ménageant un espace où se logent les parties distales des télopodites des gonopodes au repos. Cet écartement s'accompagne d'une excavation du sternite tellement profonde que celui-ci disparaît presque complètement (Fig. 6), et se réduit, médialement à un mince bandeau et, tout contre chaque hanche $(\mathbf{H})$, à une pièce subtriangulaire (st). Ces hanches se signalent en outre par la présence d'un épais prolongement distal interne (i) porteur de 4 longues soies.

Au T.VII, le gonocoele, de forme ovoïde transverse, occupe la quasi totalité de la face pleurale (Fig. 1).

Les gonopodes (Figs 2 à 4) sont très différents de ceux de l'autre espèce andalouse, $A$. cordubaensis Mauriès, 2013 [Mauriès, 2013b]. Ils s'en distinguent notamment par la sveltesse de leur télopodite (T), qui rappelle celle de A. panteli Brölemann, 1900, mais celui-ci est, comme chez cordubaensis, nettement coudé en angle presque droit. La moitié basale, érigée, constitue une hampe simple (car dépourvue de denticulations sur son bord caudal et de crête lamellaire transverse sur sa face interne). Au delà du coude (w), la moitié distale est élancée et ornée de dents; à son apex (t), elle s'orne d'une minuscule lamelle triangulaire et porte sur sa marge externe, deux grandes dents courbées vers l'apex, une proximale $(\mathbf{u})$ et une plus petite $(\mathbf{v})$ située à mi-chemin. A l'intérieur du coude, se détache un court solénomérite (s) simple et légèrement sinueux. Quant aux hanches (K), hémisphériques (côté bombé à l'extérieur et côté plan-concave à l'intérieur), elles se signalent par la présence, sur leur bord distal externe, comme chez cordubaensis, d'un lobe érigé (k). La partie préfémorale (PF), avec sa canula (= crochet coxal), n'est pas aussi nettement individualisée que chez cordubaensis.

Femelle inconnue.

Affinités. Bien que se rattachant manifestement au groupe d'espèces bético-rifaines, comme le montre la clé ci-après, la nouvelle espèce s'en distingue très nettement par ses structures gonopodiales.

\section{Clé des espèces du genre Archipolydesmus}

Cette clé est basée exclusivement sur les structures des gonopodes des mâles. Seule ne peut figurer ici $A$. 
bedeli (Brölemann, 1902), de la province de Segovia, dont le mâle est inconnu, mais qui se distingue des autres espèces par la forme ovale-fongiforme des lobes porifères paranotaux.

1. Solénomérite (branche séminale) simple ....................... 2

- Solénomérite biramé. Espèces du Nord-est de l'Espagne

2. Tiers distal du télopodite formant un angle presque droit avec les deux tiers basaux. Solénomérite situé à l'intérieur de la courbure du télopodite et non accompagné de dents ou autres aspérités. Espèces andalouses ...................... 3

- Télopodite plus ou moins arqué, non courbé en angle presque droit, sa moitié distale vaguement en raquette. Solénomérite court, situé vers la base de la partie tibiotarsale et encadré par deux ou trois aspérités plus ou moins longues. Espèces algériennes

3. Partie basale du tibiotarse en hampe érigée, grêle et droite .................................................. A. giennensis sp.n.

Espagne, prov. Jaén, troglophile.

- Partie basale du tibiotarse plus ou moins épaisse et courbée

4. Partie distale du tibiotarse ornée de deux fortes dents externes ....................... A. maroccanus (Attems, 1898)

Maroc, Tetouan.

- Partie distale du tibiotarse ornée d'une seule dent ........ 5

5. Genou du tibiotarse orné extérieurement d'une minuscule dent parfois effacée ..... A. cordubaensis Mauriès, 2013

Espagne, prov. Córdoba, troglophile.

- Genou du télopodite orné extérieurement de trois petites dent alignées ........................ A. terreus (Attems, 1952)

Espagne, prov. Cadiz, Algeciras.

6. Espèce du Parc National de Chréa .... A. chreensis Abrous-Kherbouche et Mauriès, 1996 Algérie, willaya de Blida, PN. de Chréa.

- Espèces du Parc National du Djurdjura ... A. kabylianus Abrous-Kherbouche et Mauriès, 1996 ........... A. fodili Abrous-Kherbouche et Mauriès, 1996

Algérie, willaya de Tizi-Ouzou, PN. du Djurdjura.

7. Télopodite grêle et fortement arqué …………............... 8

- Télopodite relativement robuste et faiblement arqué ........ ................................. A. ribauti (Brölemann, 1926)

France et Espagne: Catalogne.

8. Partie distale du tibiotarse (au-delà du solénomérite) courte, robuste et non arquée. A. osellai Ceuca, 1968

Espagne, prov. Huesca.

- Partie distale du tibiotarse (au-delà du solénomérite) grêle et arquée

9. Partie distale du tibiotarse (au-delà du solénomérite) pourvue de dents, son apex en crochet

A. panteli Brölemann, 1900

Espagne, prov. Cuenca.

- Partie distale du tibiotarse (au-delà du solénomérite) simple (pas de dents), longue, arquée, acuminée ... A. panteli sensu Vicente, 1981 (sp.n. ?) Espagne, prov. Tarragona (et Lérida?).

\section{CHORDEUMATIDA}

Chamaesomatidae

Caractérisés par la réduction (en tiges simples ou en épines) des présumés télopodites qui se trouvent de chaque côté et généralement à la base et en arrière du bloc gono- podial. Les deux sous-familles (Scutogoninae et Chamaesomatinae) se distinguent par les caractères externes, "néoténiques", des Chamaesomatinae: paranota latéroventrales et métazonites couverts de minuscules écailles.

\section{Scutogoninae}

Les deux tribus de cette sous-famille (Scutogonini et Origmatogonini) se distinguent par la présence, chez les Scutogonini, d'une paire de longs flagelles qui prennent naissance médialement à la base et en arrière du bloc gonopodial.

\section{Origmatogonini}

Le contenu de cette tribu nécessite quelques sérieux changements, notamment l'exclusion du genre endémique andalou Beticosoma décrit par Mauriès, 1990, du Parc Naturel de Hornachuelos (prov. Córdoba). C'est sur la base de très vagues analogies avec les gonopodes de la tribu des Origmatogonini qui nous avions été conduits à rattacher le nouveau genre à cette tribu. Cependant, au fil du temps, et compte tenu des nombreuses particularités qui nous avaient paru secondaires à l'époque, il nous apparaît aujourd'hui que ce genre doit, non seulement être exclu des Origmatogonini, mais aussi des Scutogoninae et des Chamaesomatidae, et, au-delà, prendre une place à part parmi les Chordeumatides européens, comme le sont les Striaria Bollman, 1888, parmi les Chordeumatides du nouveau Monde. Et ceci en raison des caractères particuliers observé sur les pattes antérieurs du mâle (P.3 à P.5) et notamment la présence aux P.2 d'une paire d'extraordinairement longs tractus hémipéniens...dont on ne connaît aucun équivalent chez les Chordeumatides européens. Ces caractères si particuliers peuvent constituer la matière de la diagnose d'une nouvelle famille des Beticosomatidae fam.n., dont Beticosoma est le monotype.

Les deux genres Origmatogona Ribaut, 1913, et Vascosoma Mauriès, 1966 restent d'incontestables Origmatogonini, ainsi, qu'à un degré moindre, Asturasoma Mauriès, 1981.

\section{Le genre Origmatogona Ribaut, 1913}

Représenté en Catalogne par 3 espèces, ce genre possède une espèce en Andalousie, une au Maroc et une en France:

Le type est Origmatogona catalonica Ribaut, 1913, cité des provinces catalanes de Gerona, Barcelona et Tarragona.

O. strinatii Manfredi, 1956 a été trouvé au Maroc, dans un gouffre de la région de Taza.

$O$. jacetanora Mauriès, 1964, et $O$. j. lagari Ceuca, 1988 , ont été citées des 3 provinces catalanes suscitées.

O. tinauti Mauriès, 1990 est un cavernicole de l'Andalousie de la province de Granada.

O. kimeora Mauriès, 1990 est un édaphique de France (Dordogne). 
Parmi ces 6 taxa, deux sont atypiques: O. jacetano$r a$, du fait de ses colpocoxites en oreille de lièvre et de la réduction de ses "télopodites" et surtout $O$. kimeora, qui illustre notre hypothèse [voir Mauriès \& Geoffroy, 1982] sur la condensation des pièces gonopodiales chez les petites espèces.

\section{Origmatogona toniperezi $\mathbf{s p .} \mathbf{n}$.} Figs 9-14.

Loc.type: Espagne, Andalousie, prov. Jaén, Cazorla, Cueva Secreta del Poyo Manquillo, coll. Grupo de Espeleología de Villacarillo, 31-10-2010, $1 \mathrm{O}^{\top}$ holotype, 1 \& \& 1 +j. paratypes (Musée Zoologique de Moscou).

Topotypes: mêmes collecteurs, le 12-06-2011, $3 \sigma^{7} \sigma^{7}, 4$ 우우 (Musée Zoologique de Moscou), $1 \sigma^{\gamma}, 1$ ㅇ, 1 j. (Collection Myriapodes-Onychophores du MNHN de Paris, sous le code DA 271), le 10-10-2010, 2 우, 1 j. et, le 14-04-2013, 2 juv. (Musée Zoologique de Moscou).

Nom: bien cordialement dédiée à Antonio Perez Fernandez, animateur du G.E.V.

\section{Caractères morphologiques externes}

30 anneaux, $48\left(O^{7}\right)$ ou $50(+)$ paires de pattes chez les adultes.

Coloration entièrement blanche (dépigmentation complète), pas de trace d'ocelle. Mensurations ci-après (Tableau 1).

Antennes (voir Tableau 2 ci-dessous) très longues. Massue antennaire entre 5 et 6 fois plus longue que large $\left(0,75\right.$ sur $0,13 \mathrm{~mm}$ chez le $\sigma^{7}$ holotype, 0,77 sur 0,15 chez un jeune topotype).

Vertex bombé, lisse et glabre, à sillon occipital bien marqué. Labre tridenté, rebordé par un rang de $4+$ 4 soies paralabiales.

Gnathochilarium: lames linguales pourvues chacune d'un style trifide sur leur bord distal et de trois soies: la plus longue est située dans le milieu du tiers distal de la lame, et les deux autres sont latérales et situées, l'une à mi-longueur, l'autre vers la base.

Collum en demi-lune porteur de $3+3$ macrochètes de longueur médiocre, formant de chaque côté un angle de $140^{\circ}$; les deux externes sont, de chaque côté, très marginaux, le plus interne étant nettement plus près d'une ligne sagittale imaginaire que du moyen; le rapport des distances (macrochète moyen - macrochète interne / macrochète interne-ligne sagittale imaginaire) est de $2,8 / 1$.

Corps cylindrique, dos bombé, à bourrelets paranotaux situés un peu plus haut que le milieu des flancs; les $3+3$ macrochètes, fins, longs (environ le tiers de la largeur d'un anneau moyen, plus courts sur les antérieurs, plus longs sur les postérieurs) et arqués sont portés par un minuscule cône. En vue latérale, l'externe est au milieu des flancs et se trouve seul sur la paranota. En vue dorsale, les 3 macrochètes forment de chaque côté un angle de $150^{\circ}$ sur les anneaux moyens; cet angle varie peu, augmente légèrement vers l'arrière du corps pour attteindre presque $180^{\circ}$ sur les anneaux postérieurs. Quant à la distance entre la ligne de suture (bien visible) et le macrochète interne, elle est plus importante sur les anneaux moyens que sur les antérieurs et les postérieurs: le rapport des distances ( macrochète moyen- macrochète interne / macrochète interne-sillon) est de 2,5/1 sur les anneaux antérieurs et postérieurs et de 1 / 1 sur les moyens.

Pattes ambulatoires longues (voir Tableau 3 ciaprès). Les peignes tarsaux des P.1 et P.2 ont environ une quinzaine de dents dans les deux sexes.

\section{Caractères sexuels du mâle}

Pattes antégonopodiales: P.3 à P.7 plus longues (mais pas plus robustes) que les pattes ambulatoires normales (P.12 à P.50).

Gonopodes (P.8, Figs 9-11): Construits comme chez le générotype et comme $O$. tinauti Mauriès, 1990. L'essentiel du volume du gonopode situé au-dessus du plancher est divisé transversalement en deux zones: 1) Une zone orale $(\mathbf{K a}+\mathbf{A})$ dans laquelle on distingue deux parties: une masse impaire médiane colpocoxale (Ka) échancrée en V distalement et sagittalement, couverte d'une dense et courte pilosité; cette masse est encadrée de chaque côté et caudalement à sa base par une formation vaguement triangulaire (A) sensiblement de même longueur que le masse pileuse et arbitrairement considérée comme angiocoxite; 2) une zone caudale considérée comme colpocoxale, plus large $(\mathbf{K b}+$ $\mathbf{K c}+\mathbf{K d}$ ), formant pont sagittalement au-dessus du plancher, et dans laquelle on distingue une partie médiane volumineuse et proéminente caudalement $(\mathbf{K b})$, faisant légèrement saillie distalement en une feuille (Kc) à bord distal très faiblement échancré; cette formation est flanquée de chaque côté par une masse volumineuse (Kd) aux contours boursouflés. Enfin, plus caudalement, se trouve la paire de digitations (T) caractéristiques du genre, arbitrairement considérées comme vestiges des télopodites; contrairement à celles de $O$. tinauti, elles se rétrécissent progressivement de leur base, qui est large, à leur apex, qui est étroit et émoussé.

Paragonopodes (P.9, Fig. 12) se distinguant de ceux de $O$. tinauti par le préfémur plus claviforme, par l'absence d'un bombement évident de sa moitié distale, et par la nettement plus grande longueur de l'expansion coxale effilée.

Pattes post-gonopodiales (P.10 et P.11) à sacs coxaux; des cornes coxales postérieures seulement aux P.11 (Fig. 13). Le métatarse des P.11 est notablement plus court que sur les autres paires (Tableau 3 ).

\section{Caractères sexuels de la femelle}

La répartition des macrochètes est, sur tous les anneaux, comme chez le $\sigma^{7}$ holotype.

Les vulves (Fig. 14) sont très semblables à celles de $O$. tinauti; elles sont étroitement serrées l'une contre l'autre. Elles sont dissymétriques du fait de la position et du trajet en courbe de la gouttière aposématique (ga). La valve interne (vi), qui est plus courte que la valve externe (ve) est prolongée caudalement, comme chez $O$. tinauti, par une longue corne; mais ici cet appendice est faiblement arqué ou droit au lieu d'être fortement coudé. L'opercule (op) est très discret, à peine visible en vue distale.

Affinités évidentes avec $O$. tinauti, dont il se distingue par des détails des structures gonopodiales. Une 

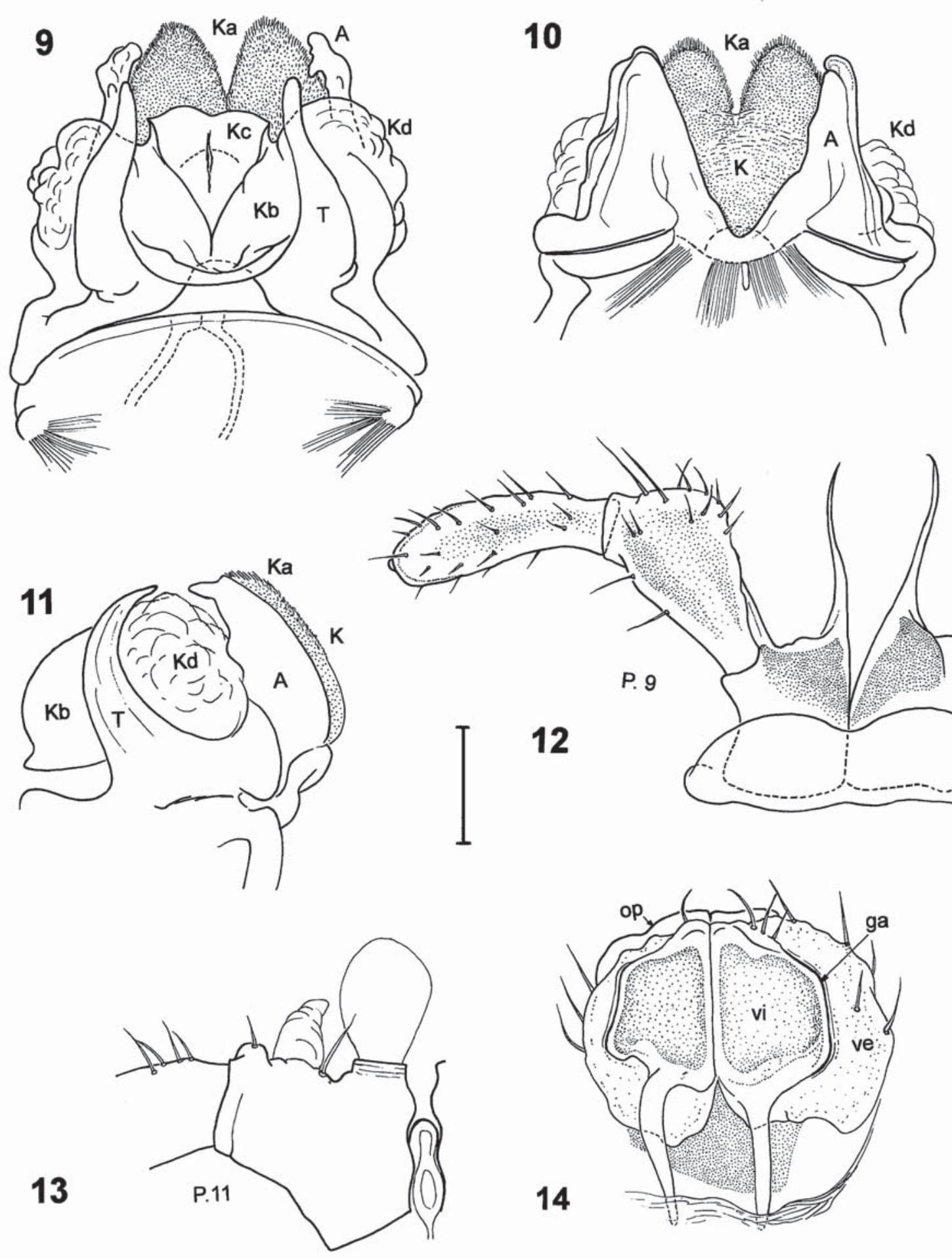

Figs 9-14. Origmatogona toniperezi sp.n., holotype $\sigma^{7}$ et 9 allotype: 9 - vue caudale du bloc gonopodial; 10 — vue orale du même; 11 - vue latérale du côté gauche du même; 12 - paragonopodes, face orale; 13 - hanche des P.11, face orale; 14 — vue distale des deux vulves. Echelle $0,1 \mathrm{~mm}$.

Figs 9-14. Origmatogona toniperezi sp.n., holotype $\sigma^{7}$ and 9 allotype: $9-$ caudal view of gonopodial block; $10-$ oral view of the same; 11 - lateral view of left side of the same; 12 - paragonopods, oral view; 13 - coxa 11, oral view; 14 - distal view of both vulvae. Scale bar $0.1 \mathrm{~mm}$.

Рис. 9-14. Origmatogona toniperezi sp.n., О голотип и аллотип +: 9 - гоноподиальный блок, сзади; 10 - то же, спереди; 11 то же, вид сбоку левой стороны; 12 - парагоноподы, спереди; 13 - тазик 11 , спереди; 14 - дистальный вид обеих вульв. Масштаб 0,1 мм. 
Tableau 1. Mensurations (en $\mathrm{mm}$ ) des types et de quelques topotypes. L — longueur du corps; N — nombre d'anneaux; ltj — largeur de la tête (joues comprises); lc — largeur du collum; lm — largeur du métazonite; lp — largeur du prozonite; sp — section; L / 1 - longueur sur largeur de la massue antennaire.

Table 1. Measurements (in $\mathrm{mm}$ ) of the types and some topotypes.

$\mathrm{L}$ — body length; $\mathrm{N}$ — number of segments; $1 \mathrm{tj}$ — width of head (including genae); $1 \mathrm{c}$ — width of collum; $1 \mathrm{~m}$ — width of metazonite; $1 \mathrm{p}$ - width of prozonite; sp - section; L/1 - length to width ratio of antennal club.

Таблица 1. Промеры (в мм) типовых и нетиповых экземпляров.

$\mathrm{L}$ — длина тела; $\mathrm{N}$ — число сегментов; $\mathrm{ltj}$ — ширина головы (включая щеки); $1 \mathrm{c}$ — ширина коллума; $\operatorname{lm}$ — ширина метазонита; $1 \mathrm{p}$ - ширина прозонита; $\mathrm{sp}$ - поперечник; L/1 - соотношение длины и ширины булавы усиков.

\begin{tabular}{|c|c|c|c|c|c|c|c|c|c|}
\hline & \multirow{2}{*}{$\begin{array}{l}\text { date } \\
\text { récolte }\end{array}$} & \multirow{2}{*}{$\mathrm{N}$. } & \multirow{2}{*}{ L. } & \multirow{2}{*}{ 1.t.j. } & \multirow{2}{*}{ 1.c. } & \multicolumn{2}{|c|}{ anneaux moyens } & \multirow{2}{*}{$\begin{array}{c}\begin{array}{c}\text { anneaux } \\
\text { postérieurs }\end{array} \\
\text { s.p. }\end{array}$} & \multirow{2}{*}{$\begin{array}{c}\text { massue antennaire } \\
\text { L. } / 1 .=\end{array}$} \\
\hline & & & & & & 1.p. - s.p. & 1.m. & & \\
\hline$\sigma^{2}$ holotype & \multirow{3}{*}{$\begin{array}{c}31-10 \\
2010\end{array}$} & 30 & 8,3 & 0,84 & 0,69 & 0,71 & 0,86 & 0,47 & 5,8 \\
\hline$q$ allotype & & 30 & 9,0 & 0,84 & $?$ & 0,74 & 0,98 & 0,64 & 5,3 \\
\hline q j. paratype & & 28 & 8,4 & 1,01 & 0,70 & 0,64 & 0,90 & 0,55 & 5,8 \\
\hline$\sigma^{7}$ topotype & \multirow{4}{*}{$\begin{array}{c}12-06 \\
2011\end{array}$} & 30 & 8,5 & 1,00 & 0,18 & 0,72 & 1,18 & 0,63 & 5,2 \\
\hline O topotype & & 30 & 9,6 & - & - & 1,04 & 1,24 & 0,62 & 5,7 \\
\hline q topotype & & 30 & 9,8 & - & - & 0,83 & 0,99 & 0,60 & 5,5 \\
\hline o j. topotype & & 28 & 7,24 & 0,80 & 0,60 & 0,77 & 0,87 & 0,60 & 5,1 \\
\hline topotype & \multirow{3}{*}{$\begin{array}{l}10-10 \\
2010\end{array}$} & 30 & 10,1 & 1,01 & $?$ & 0,66 & 1,18 & 0,59 & 5,4 \\
\hline q topotype & & 30 & 8,2 & - & - & - & - & - & - \\
\hline q topotype & & 30 & 7,9 & 1,03 & 0,63 & 0,72 & 1,09 & 0,64 & 5,3 \\
\hline juv. topotype & \multirow{2}{*}{$\begin{array}{l}14-04 \\
2013\end{array}$} & 23 & 4,9 & & & & & & \\
\hline juv. topotype & & 19 & 2,7 & & & & & & \\
\hline
\end{tabular}

Tableau 2. Longueur (en $\mathrm{mm}$ ) des antennes et des antennomères. Table 2. Length (in $\mathrm{mm}$ ) of antennae and antennomeres. Таблица 2. Длина (в мм) усиков и отдельных их члеников.

\begin{tabular}{|c|c|c|c|c|c|c|c|c|c|}
\hline & date. & $\mathrm{L}$ & 1 & 2 & 3 & 4 & 5 & 6 & $7+8$ \\
\hline$\sigma^{7}$ holotype & \multirow{3}{*}{$\begin{array}{c}31-10 \\
2010\end{array}$} & 1,62 & 0,09 & 0,18 & 0,42 & 0,24 & 0,45 & 0,14 & 0,16 \\
\hline$q$ allotype & & 1,63 & 0,07 & 0,22 & 0,46 & 0,23 & 0,47 & 0,19 & 0,17 \\
\hline o j. paratype & & 1,52 & 0,06 & 0,15 & 0,39 & 0,18 & 0,41 & 0,16 & 0,15 \\
\hline$q^{9}$ j. topotype & $12-06-11$ & 1,45 & 0,11 & 0,18 & 0,41 & 0,18 & 0,43 & 0,18 & 0,16 \\
\hline
\end{tabular}

Tableau 3. Longueur $(\mathrm{mm})$ de quelques pattes du $\sigma^{\top}$ holotype $\&$ de leurs podomères. Table 3. Length (in $\mathrm{mm}$ ) of several legs in the $\sigma^{7}$ holotype $\&$ of their podomeres.

Таблица 3. Длина (в мм) нескольких ног у голотипа $\sigma^{7}$ и их отдельных члеников.

\begin{tabular}{|l|c|c|c|c|c|c|c|c|c|}
\hline & Longueur & $\begin{array}{c}\text { coxa }+ \\
\text { trochanter }\end{array}$ & préfémur & fémur & tibia & tarse & métatarse & uncus & $\begin{array}{l}\text { peigne du } \\
\text { métatarse }\end{array}$ \\
\hline P. 1 & 0,79 & 0,09 & 0,23 & 0,08 & 0,07 & \multicolumn{2}{|c|}{0,28} & 0,04 & 15 dents \\
\hline P. 2 & 0,93 & 0,18 & 0,22 & 0,10 & 0,08 & & 0,30 & 0,05 & 15 dents \\
\hline P. 7 & 1,42 & 0,14 & 0,27 & 0,32 & 0,11 & 0,10 & 0,42 & 0,06 & 0 \\
\hline P. 11 & 0,98 & 0,19 & 0,23 & 0,24 & 0,08 & 0,07 & 0,14 & 0,03 & 0 \\
\hline P. 28 & 1,21 & 0,16 & 0,21 & 0,30 & 0,07 & 0,07 & 0,34 & 0,06 & 0 \\
\hline
\end{tabular}

clé des espèces du genre se trouve dans Mauriès [1990: 52].

\section{Opisthocheiridae}

\section{Le genre Ceratosphys Ribaut, 1920, en Andalousie}

Le genre Ceratosphys Ribaut, 1920, qui a fait, depuis Mauriès [1990b], l'objet de notes récentes [Mauriès, 2013a, b] était connu jusqu'à ce jour en Andalousie par 5 espèces; deux nouvelles sont décrites ci-après; la présence de $C$. solanasi en Andalousie même est mise en doute:

1) C. hispanica Ribaut, 1920, citée par son auteur comme type de son sous-genre Haplosphys, a été oubliée par mégarde et n'a été décrite que plus tard par Mauriès [1968]. Elle a été récoltée en la prov. de Cádiz, à Jerez de la Frontera, dans la Cueva de las Motillas. 
2) C. nodipes (Attems, 1952), redécrite par Mauriès [1978], provient de la prov. de Málaga, en la Sierra Ronda, au Monte Arastepa (recte Jarastepar), à $10 \mathrm{~km}$ au sud de Ronda, vers $1400 \mathrm{~m}$ d'altitude. Une forme affine a été récoltée en 2002 à $20 \mathrm{~km}$ de là, et récemment citée [Mauriès, 2013b] en la Sima del Republicano, à Villaluenga del Rosario (prov. Cádiz),

3) C. soutadei Mauriès, 1970, a été récoltée par l'auteur dans la Sierra Nevada (Granada) au Barranco de Veleta, à $2800 \mathrm{~m}$ d'altitude.

4) C. deharvengi Mauriès, 1978, de la prov. de Màlaga, a été récoltée par Louis Deharveng dans la Sierra de las Nieves, à $20 \mathrm{~km}$ au sud-est de Ronda, vers 1600-1900 m. d'altitude.

5) C. solanasi Mauriès et Vicente, 1977, espèce type de Proceratosphys Mauriès et Vicente, 1977, décrit comme sous-genre et dont le statut au rang de genre est contestable, n'a pas été décrite d'Andalousie, mais de la Cueva de los Chorros, qui se trouve dans une zone toute proche, dans la province d'Albacete, à Riopar. Récemment retrouvée dans la même grotte et dans la grotte voisine del Farallón, cette espèce a été citée récemment d' Andalousie, dans la prov. de Jaén, en la Cueva Secreta del Poyo Manquillo, à Cazorla, par Golovatch \& Mauriès [2013]. En l'absence de mâle, ces exempaires qui présentent des vulves presque identiques à celles de $C$. solanasi ont été identifiés comme tels. Or, il s'avère que les femelles de l'une des deux espèces nouvelles décrites ici même, $C$. fernandoi sp.n. a des vulves quasiment identiques à celles de $C$. solana$s i$ ! Il y a donc de très fortes probabilités pour que les femelles du Poyo Manquillo appartiennent en fait, en raison de leur proximité géographique, à l'une des deux espèces décrites ci-après des zones voisines de Cazorla (C. flammeola sp.n.) et de Castril (C. fernandoi sp.n.). Il reste que le choix entre ces deux taxa ne peut pour le moment se faire, la femelle de $C$. flammeola étant encore inconnue. La présence de $C$. solanasi en Andalousie même reste donc douteuse et à établir.

6) C. jabaliensis Mauriès, 2013, est la première espèce du genre signalée dans la zone des Parcs naturels des Sierras de Cazorla, Segura y Las Villas: prov. Jaén, Pontones, Cueva del Jabalí. Elle se distingue nettement des deux nouvelles espèces (ses voisines géographiques) mais aussi de toutes les espèces du genre par l'extrême simplicité des télopodites de ses gonopodes.

\section{Ceratosphys flammeola sp.n.} Figs 15-21.

Loc. type: Espagne, prov. Jaén, Cazorla, Cueva Moreno, 1404-2013, coll. Grupo de Espeleología de Villacarillo, $\sigma^{7}$ holotype, déposé au Musée Zoologique de Moscou.

Nom: terme latin désignant la coloration orangée.

\section{Caractères morphologiques externes}

30 anneaux chez les adultes des deux sexes. Coloration brunâtre claire, alternée de blanc (aux prozonites) et d'orange (aux métazonites).

Yeux composés de 22 ocelles disposés en 8 rangs: 11123455 (œil gauche) et 11233345 (œil droit) inclus dans un triangle brun-noir. Vertex très légèrement concave. Labre tridenté orné de $6+6$ soies paralabiales.

Antennes pas particulièrement longues, deux fois plus longues que la largeur de la tête, joues comprises.

Mensurations (en $\mathrm{mm}$ ):

Longueur du corps: 8,7 .

Largeur de la tête (joues comprises): 0,72; du collum: 0,61 .

Largeur du métazonite du $5^{\text {ème }}$ anneau (T. V): 0,83.

Anneau moyen: largeur du prozonite: 0,86 ; diamètre vertical: 0,80 ; largeur du métazonite: 0,92 ; diamètre vertical: 0,87 .

Longueur théorique de l'antenne ( $\mathrm{mm}): 1,64=0,07$ $+0,20+0,46+0,23+0,37+0,17+0,14$. Longueur réelle: 1,56. Massue antennaire: 4,5 fois plus longue que large.

Longueur des P.7 et P.10: 1,09 mm. L'uncus des P. 11 mesure $0,05 \mathrm{~mm}$. Il est flanqué d'une fine épine accessoire plus longue que lui.

Collum en demi-lune, avec $3+3$ macrochètes dont le plus externe, de chaque côté, est complètement marginal.

Autres anneaux: les paranota sont médiocres, situées un peu plus haut que le milieu des flancs, leur bord latéral paraissant, en vue dorsale, légèrement arqué, avec des angles émoussés, surtout l'antérieur. La faible longueur de chaque paranota est la conséquence de la présence d'un sillon (ou d'une rigole) dite oblique (parce que ce sillon, presque rectiligne, apparaît, en vue latérale, comme incliné, plus haut oralement que caudalement). Ce sillon oblique isole chaque paranota du reste du dos, le faisant apparaître plus comme un simple bourrelet que comme une expansion aliforme. De chaque côté, les deux macrochètes les plus externes se trouvent à l'extérieur de ce sillon, sur le bord du bourrelet paranotal, l'un (le moyen) situé en avant, l'autre (l'externe) en position postérieure. Ces $3+3$ macrochètes, courts et fins, forment de part et d'autre du sillon dorso-médian, un angle de 95 à $100^{\circ}$ sur les anneaux moyens, de 110 à $120^{\circ}$ sur les postérieurs; le macrochète interne est un peu plus proche du moyen que du sillon dorso-médian sur les anneaux moyens, mais à égale distance sur les anneaux postérieurs.

\section{Caractères sexuels du mâle}

Pattes antégonopodiales: P.3 à P.7 plus longues et plus robustes que les P.1 et P.2, comme chez tous les chordeumatides, mais pas plus longues ni plus robustes que les autres pattes ambulatoires. Aux P.7, les hanches sont pourvues d'une courte digitation distale sur leur face caudale, et le métatarse porte seulement quelques papilles sur la face interne de son tiers distal. Uncus comme sur les pattes postgonopodiales.

Gonopodes (Figs 15-17): Constitués, comme chez ses congénères de trois éléments principaux: d'un synangiocoxite (A) antéro-basal discret et de forme variable, d'un syncolpocoxite (K) en écu ou bouclier qui ferme la face postérieure des gonopodes, et, de chaque côté, un télopodite (T) plus ou moins biramé et souvent arqué. 


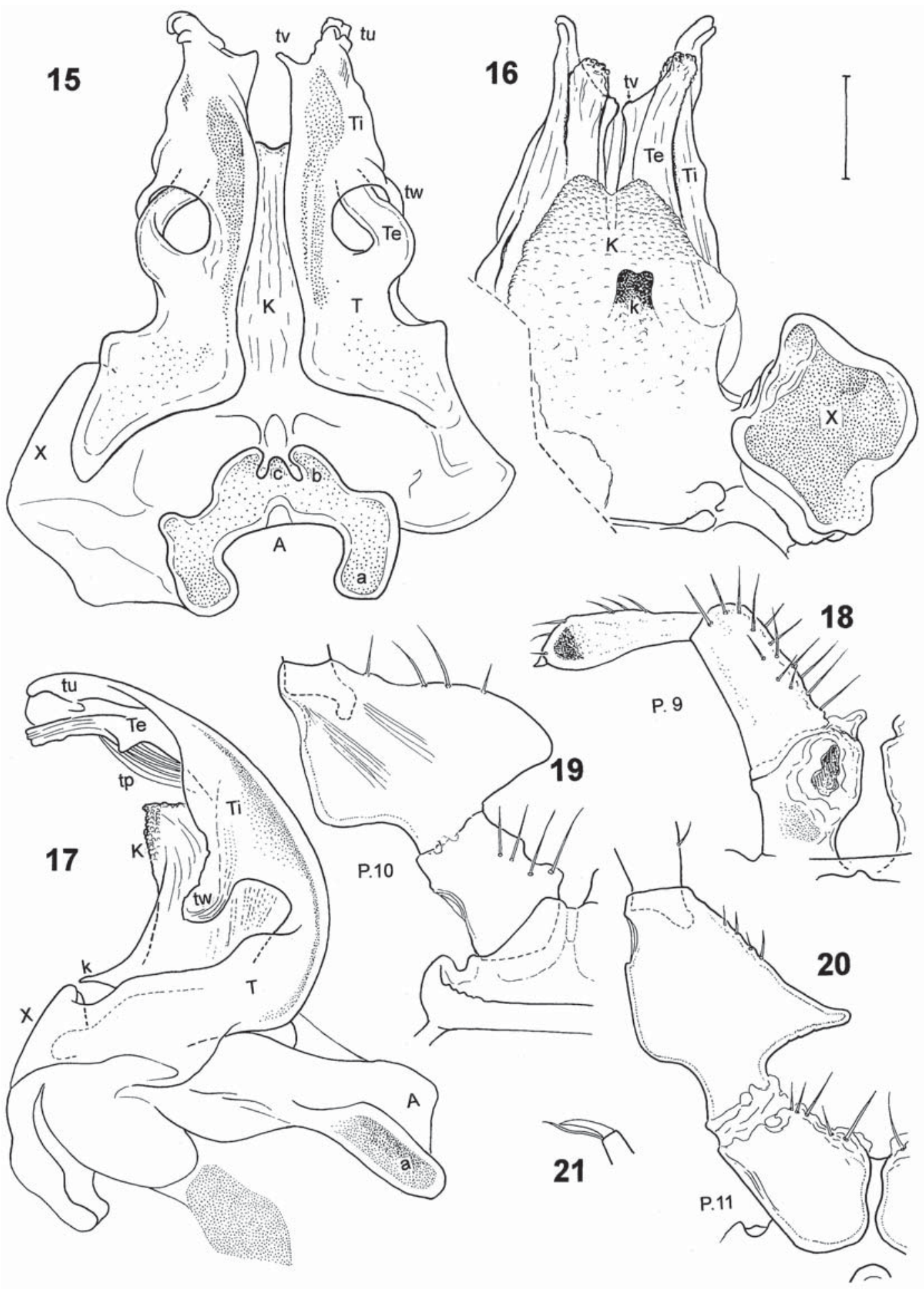

Figs 15-21. Ceratosphys flammeola sp.n., holotype: 15 — vue orale du bloc gonopodial; 16 — vue caudale du même; 17 — vue latérale externe du côté gauche du même; 18 - paragonopodes; 19 - hanche et préfémur d'une P.10, face orale; 20 — hanche et préfémur d'une P.11, face caudale; 21 - uncus d'une P.11. Echelle 0,1 mm.

Figs 15-21. Ceratosphys flammeola sp.n., holotype: 15 - oral view of gonopodial block; 16 - caudal view of the same; 17 - lateral external view of left side of the same; 18 - paragonopods; 19 - coxa and prefemur of leg 10, oral view; 20 - coxa and prefemur of leg 11, caudal view; 21 - claw of leg 11 . Scale bar $0.1 \mathrm{~mm}$

Рис. 15-21. Ceratosphys flammeola sp.n., голотип: 15 - вид спереди гоноподиального блока; 16 - сзади то же; 17 - вид сбоку снаружи левой стороны того же; 18 - парагоноподы; 19 - тазик и предбедро 10 , спереди; 20 - тазик и предбедро 11 , сзади; 21 - коготок ноги 11. Масштаб 0,1 мм. 
Cependant, ces gonopodes se rattachent manifestement à ceux du quatuor d'espèces bético-rifaines $(C$. nodipes (Attems, 1952), C. soutadei Mauriès, 1970, C. deharvengi Mauriès, 1978, C. maroccana Mauriès, 1985) qui peuple les zones montagneuses des provinces de Granada et de Màlaga ainsi que le Moyen-Atlas marocain. Ils en possèdent les deux principales caractéristiques:

1) un synangiocoxite $(\mathbf{A})$ constitué par une paire de masses plus ou moins soudées sagittalement, mais qui le sont ici largement et qui émettent, de chaque côté, un lobe basal arrondi (a) et deux petits lobes distaux (b) qui encadrent un plus petit lobe médian (c)

2) une paire de télopodites (T) qui, comme chez trois des espèces du quatuor sus-nommé (nodipes, deharvengi et maroccana), se divisent à leur mi-longueur en deux rameaux (Ti \& Te) qui s'entrecroisent distalement de telle sorte que le plus grêle, l'externe (Te) passe sous la protection du plus robuste, l'interne (Ti), qui de ce fait devient externe, tout en s'élargissant notablement; en même temps, il émet distalement un lobe allongé externe (tu), un angle interne (tv), et, sur son bord externe, et bien plus basalement, une forte digitation rétroarquée (tw). Quant au rameau externe, presque entièrement caché, il montre en vue latérale une longue lame striée longitudinalement (tp), ces stries ayant l'apparence de lanières. L'embase de chaque télopodite («talon du cheirite» selon Ribaut [1920]) se présente comme une puissante plaque $(\mathbf{X})$ située en position postérieure et latérale sur le côté du syncolpocoxite.

Quant au syncolpocoxite (K), il se singularise par la présence, au milieu de sa face caudale, par l'émergence d'un très court prolongement impair $(\mathbf{k})$ à bord distal bilobé; sa face caudale n'est pas ornée, et il ne se distingue des espèces du quatuor bético-rifain que par son bord distal simple (non rebordé comme chez nodipes et soutadei) et peu échancré en V très ouvert.

Paragonopodes (Fig. 18) construits comme chez les quatre bético-rifaines et comme $C$. solanasi Mauriès et Vicente, 1977: membre tri-articulé constitué d'une hanche et d'un télopodite bi-articulé. Ce dernier comprend un préfémur simple, subcylindrique, deux fois plus long que large (et deux fois plus long que la hanche) à l'extrémité duquel s'articule le podomère terminal claviforme qui forme avec lui un angle de $120^{\circ}$ environ.

Pattes postgonopodiales (Figs 19-21): Les P.10 et les P.11 n'ont de papilles métatarsales que sur la moitié distale de leur face ventrale; les déformations du préfémur sont très semblables à celles observées chez C. deharvengi: un grand lobe arrondi interne aux P.10 (Fig. 19), un lobe interne acuminé rétroarqué et un léger renflement externe aux P.11 (Fig. 20). L'uncus (Fig. 21) est relativement grêle et acuminé, et flanqué d'une soie accessoire plus longue que lui.

Femelle inconnue.

Affinités incontestables avec les quatre espèces bético-rifaines, plus particulièrement avec $C$. nodipes, $C$. deharvengi et $C$. maroccana qui possèdent une paire de télopodites gonopodiaux divisés chacun en deux branches entrecroisées; un peu différent est $C$. soutadei, chez qui les télopodites ne sont pas divisés en deux branches mais possèdent comme les trois autres espèces un petit rameau externe rétroarqué.

\section{Ceratosphys fernandoi sp.n.}

Figs 22-31.

Loc. type: Espagne, prov. Granada, Castril, Cueva de Don Fernando, coll. Grupo de Espeleología de Villacarillo, 02-05-2009; $1 \sigma^{7}$ holotype, $1+$ allotype, $1 \sigma^{7}, 1+$ préadulte, 2 juv. (stades VI \& VII) paratypes, sont déposés au Musée Zoologique de Moscou, 2 $\sigma^{7} \sigma^{7}$ et 2 juv. (stade VII) paratypes sont déposés dans la collection Myriapodes-Onychophores du MNHN de Paris, sous le code DA 270.

Nom: dérivé du nom de la grotte.

\section{Caractères morphologiques externes}

Coloration générale brunâtre claire (dorsalement et aux antennes), encore plus claire ventralement, mais plus foncée sur la tête et à l'extrémité du telson; pattes blanchâtres.

Tête: Vertex plan-concave chez les $\sigma^{\gamma}$, convexe chez les $q$ et les jeunes. Labre tridenté, encadré de chaque côté par 6/7 soies paralabiales. Yeux inscrits dans un champ pigmenté noir vaguement pyriforme, ayant chez les adultes de 15 à 20 ocelles répartis sur 7 ou 8 rangs, et, chez les jeunes des stades VI et VII, de 12 à 14, répartis sur 6 rangs (voir tableau 4 ci-dessous). La répartition des ocelles dans chaque rang et le nombre de rangs ne permettent pas d'établir quelque corrélation avec les stades de développement, comme cela est possible chez la plupart des chordeumatides.

Antennes: antenne droite du $\sigma^{7}$ paratype et celle de la + allotype mesurant 1,60 mm. Massue antennaire 5 fois plus longue que large chez le $\sigma^{\top}$ holotype et chez un $O^{7}$ paratype, 4,5 fois chez la $q$ allotype.

Paranota médiocres, comme chez l'espèce précédente, en bourrelets, du fait de la présence de chaque côté du métazonite, du sillon dit oblique qui les sépare du reste du dos. Les 3 macrochètes forment entre eux, en apparence, dans une vue dorsale, de chaque côté, un angle de $95^{\circ}$ environ. Sur les anneaux moyens, la distance qui sépare les deux externes est égale à celle qui sépare l'interne du moyen. Quant au macrochète interne, il est plus proche du moyen que du sillon dorso-médian, dans le rapport $1 /$ 1,5. Sur les anneaux postérieurs, seule change la distance entre les deux externes; celle-ci va diminuant sensiblement, jusqu'à diminuer de moitié sur les derniers anneaux, du fait de l'effacement progressif des paranota.

Les macrochètes sont très fins, d'une longueur médiocre, à peu près égale à la largeur d'une paranota d'un anneau moyen; ils sont plus longs sur les anneaux postérieurs.

Pattes ambulatoires du milieu du corps mesurant à peine plus que la largeur d'un métazonite.

\section{Caractères sexuels du mâle}

Pattes antégonopodiales: en arrière des P.2, les P.3 à P.7 sont robustes, épaisses, seules les P.7 sont un peu plus faibles et leur hanches pourvues d'une longue digitation caudale (Fig. 26). 

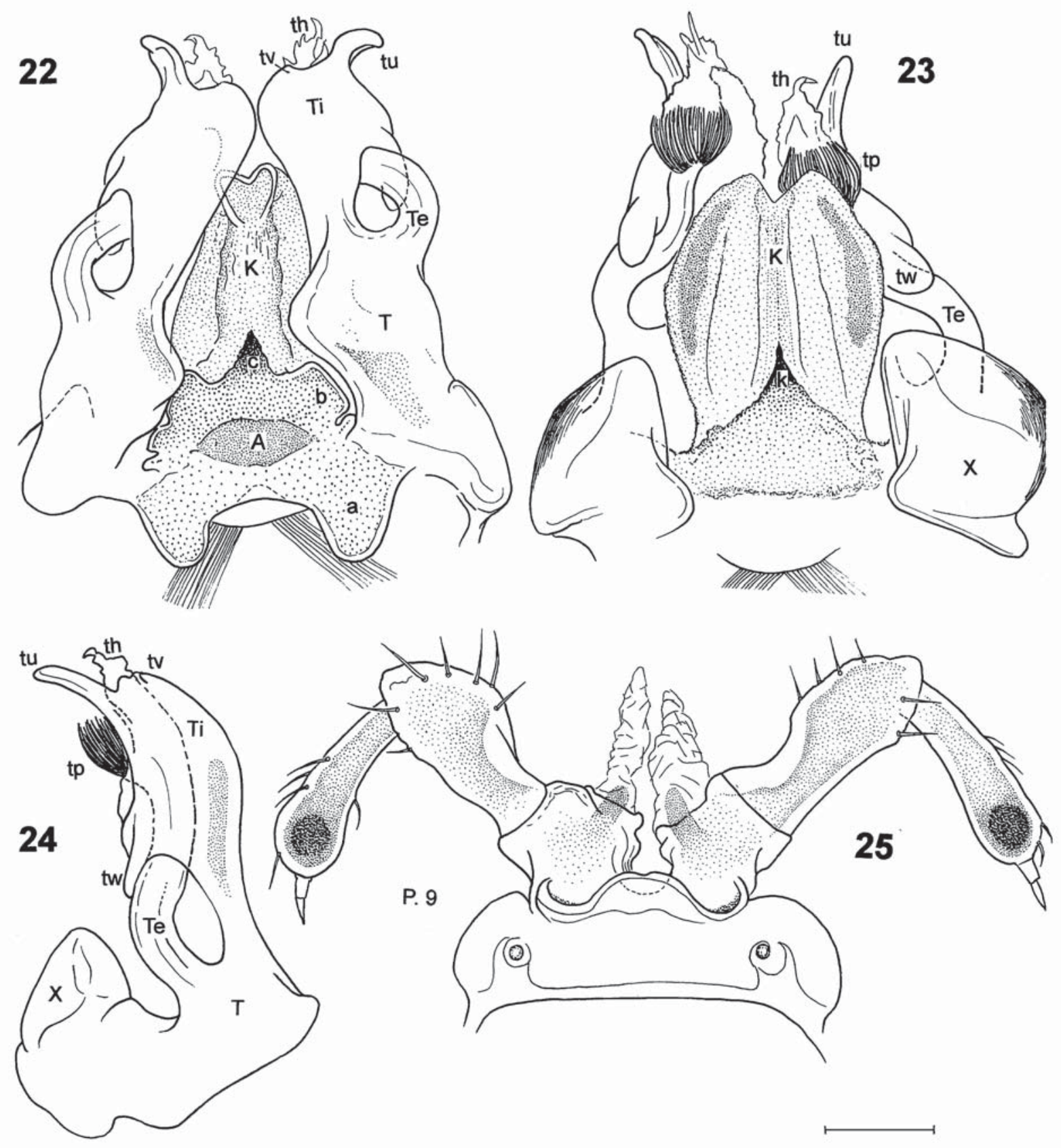

Figs 22-25. Ceratosphys fernandoi sp.n., holotype: 22 - vue orale du bloc gonopodial; 23 — vue caudale du même; 24 - vue latérale externe du côté gauche du même; 25 - paragonopodes, face orale. Echelle $0,1 \mathrm{~mm}$.

Figs 22-25. Ceratosphys fernandoi sp.n., holotype: 22 - oral view of gonopodial block; 23 - caudal view of the same; 24 - lateral external view of left side of the same; 25 - paragonopods, oral view. Scale bar $0.1 \mathrm{~mm}$.

Рис. 22-25. Ceratosphys fernandoi sp.n., голотип: 22 - вид спереди гоноподиального блока; 23 - то же, сзади; 24 - то же, вид сбоку снаружи левой стороны; 25 - парагоноподы, спереди. Масштаб 0,1 мм.

Gonopodes (Figs 22-24): très semblables à ceux de l'espèce précédente et ne s'en distinguant que par les détails suivants:

1) Synangiocoxite (A) plus massif, les deux lobes inférieurs (a) plus courts et les 3 lobes distaux (b et $\mathbf{c}$ ) peu individualisés.
2) Télopodites $(\mathbf{T})$ présentant aussi un «talon» caudal (X) volumineux, une division de la partie moyenne en deux branches (Ti \& $\mathbf{T e}$ ) dont l'interne (Ti) enveloppe l'autre (Te) plus distalement en la dissimulant partiellement. La partie distale de la branche interne est simple et large; son bord distal est en large lobe 
Tableau 4. Longueur de chaque exemplaire $(\mathrm{mm}) \&$ nombre d'ocelles. Table 4. Length of each specimen (in $\mathrm{mm}$ ) \& number of ocelli. Таблица 4. Длина кадого экземпляра (в мм) и число глазков.

\begin{tabular}{|c|c|c|c|}
\hline & \multirow{2}{*}{$\begin{array}{l}\text { Longueur } \\
\text { en mm }\end{array}$} & \multicolumn{2}{|c|}{ Nombre d'ocelles par rang } \\
\hline & & œil gauche & œil droit \\
\hline$\sigma^{7}$ holotype & 9,2 & $15: 1112343$ & $17: 1223333$ \\
\hline$\sigma^{7}$ paratype (disséqué) & 8,8 & 15: 1112343 & 20: 11223443 \\
\hline autre $\bigcirc^{7}$ paratype disséqué & 8,8 & & \\
\hline autre $\sigma^{7}$ paratype non disséqué & 8,9 & & \\
\hline q allotype & 9,6 & 18: 1223442 & 18: 11123442 \\
\hline q préadulte paratype & 8,6 & & \\
\hline juv. stade VII paratype & 6,6 & 14: 112343 & \\
\hline juv. stade VII paratype & 6,2 & 12: 112233 & \\
\hline juv. stade VII paratype & 6,0 & 11: 111233 & \\
\hline juv. stade VI paratype & 5,7 & 12: 112233 & \\
\hline
\end{tabular}

Tableau 5. Mensurations ( $\mathrm{mm}$ ) d'un $\Im^{\top}$ paratype et de la + allotype. Table 5. Measurements (in $\mathrm{mm}$ ) of a $\sigma^{7}$ paratype and the + allotype. Таблица 5. Размеры (в мм) паратипа самца и аллотипа самки.

\begin{tabular}{|l|c|c|}
\hline & un O $^{7}$ paratype & + allotype \\
\hline Largeur de la tête (joues comprises) & 0,80 & 0,76 \\
\hline Largeur du collum: & 0,63 & \\
\hline Largeur du 5 ${ }^{\text {eme }}$ anneau (T.V): & 0,78 & 0,71 \\
\hline Largeur prozonite du T.VI & & 0,88 \\
\hline Largeur métazonite du T.VI & & 0,76 \\
\hline Largeur prozonite anneau moyen (T.XIV) & 0,76 & 0,93 \\
\hline Largeur métazonite anneau moyen (T.XIV) & 0,92 & 0,77 \\
\hline Diamètre vertical anneau moyen (T.XIV) & 0,74 & 0,60 \\
\hline Section anneau postérieur (T.XXVI) & 0,64 & \\
\hline
\end{tabular}

arrondi du côté interne (tv) et se prolonge latéralement par une simple corne courbée vers l'extérieur (tu). Quant à la partie rétroarquée (tw), elle est ici nettement plus discrète que chez l'espèce précédente. La branche externe possède un faisceau de lanières (tp) en très court et large pinceau et se termine par une lame hyaline très découpée (th).

3) Quant au syncolpocoxite, il possède aussi, sur sa face caudale, un petit prolongement impair (k) qui est ici en pointe et non bilobé et, sur sa face orale, une petite formation distale en forme de cœur (Fig. 22) qui souligne son échancrure en $\mathrm{V}$ ouvert.

Pattes antégonopodiales (P.3-P.7) robustes; hanches des P.7 (Fig. 26) pourvues d'une longue digitation érigée.

Paragonopodes (Fig. 25) très semblables à ceux de $C$. solanasi et $C$. soutadei, à membres triarticulés composés chacun d'une hanche (coxa) pourvue d'un sac coxal membraneux ici évaginé en manchon, et d'un télopodite biarticulé; ce dernier se compose d'un préfémur dont la moitié basale de son profil interne est largement et peu profondément échancrée, et d'un podomère pyriforme (fémur) qui se détache en angle droit de l'extrémité du préfémur. Le reste du télopodite est représenté par une longue épine rélictuelle plantée à l'apex du fémur.

Pattes postgonopodiales un peu moins robustes que les P.3-P.7. Les P.10 et P.11 le sont encore un peu moins que les ambulatoires qui les suivent, leurs coxae sont pourvues de sacs coxaux, et leurs préfémurs sont volumineux et ornés d'une corne interne (Figs 27 et 28).

\section{Caractères sexuels de la femelle}

Les vulves (Figs 29-31) sont construites comme chez $C$. solanasi et $C$. jabaliensis: chaque bourse, courte et symétrique est protégée oralement par un opercule (op) court et large qui est surmonté par un fort prolongement supra-operculaire (sop) incliné au-dessus du cimier; elle est accompagnée caudalement par un organe post-vulvaire (pv). Ces vulves se distinguent néanmoins comme suit:

L'opercule est court et enveloppant, porteur de 2 paires de longues soies situées très latéralement; son prolongement médian, l'organe supra-operculaire (sop) n'a pas ici la forme en raquette de $C$. jabaliensis et de $C$. solanasi, ni la forme vaguement cruciforme de $C$. hispanica, mais se présente comme une expansion allongée membraneuse qui a été accidentellement coupée sur la vulve droite, mais a subsisté sur la vulve gauche (Fig. 31).

L'organe post-vulvaire, qui se montre sous divers aspects selon l'angle de vision (Figs 29, 30 et 31) apparaît Fig. 30 comme une longue corne acuminée qui s'incline vers l'avant au-dessus du cimier de la bourse, comme chez $C$. solanasi. 

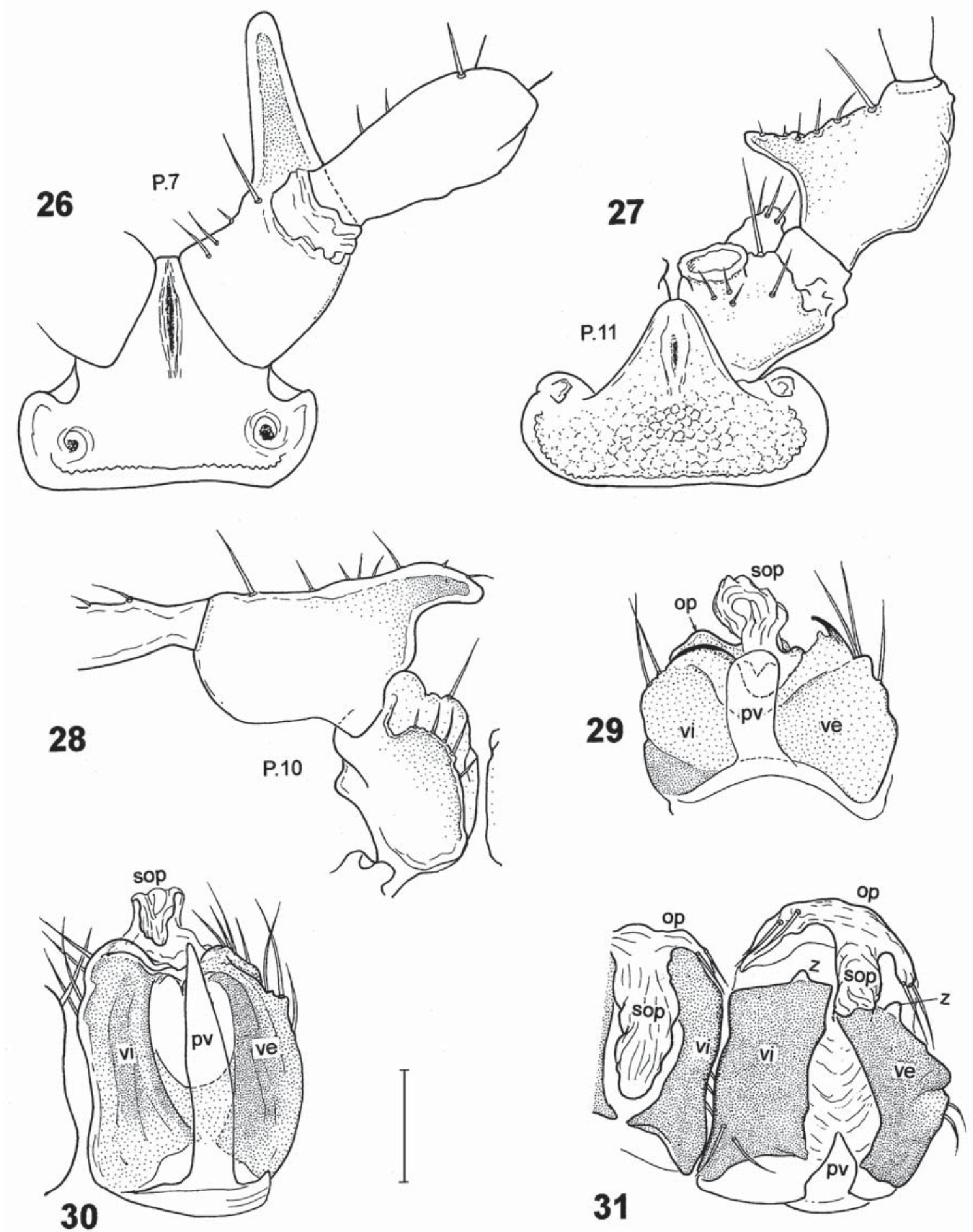

Figs 26-31. Ceratosphys fernandoi sp.n., holotype $\sigma^{7}$ et 9 allotype: 26 - base de la P.7 (sternite, hanche et préfémur), face orale; 27 - P.11 (sternite, hanche et préfémur), face orale; 28 - P.10 (hanche et préfémur), face caudale; 29 - vulve droite, face postérieure; 30 - la même, vue distale; 31 - la même, avec une partie de la gauche, en vue antéro-distale. Echelle $0,1 \mathrm{~mm}$.

Figs 26-31. Ceratosphys fernandoi sp.n., holotype $\sigma^{7}$ and 9 allotype: 26 - base of leg P.7 (sternite, coxa and prefemur), oral view; 27 - leg 11 (sternite, coxa and prefemur), oral view; 28 - leg 10 (coxa and prefemur), caudal view; 29 - right vulva, caudal view; 30 the same, distal view; 31 - the same, partly together with the left one, anterodistal view. Scale bar $0.1 \mathrm{~mm}$.

Рис. 26-31. Ceratosphys fernandoi sp.n., O голотип and аллотип ㅇ: 26 - основание ноги 7 (стернит, тазик и предбедро), спереди; 27 - нога 11 (стернит, тазик и предбедро), спереди; 28 - нога 10 (тазик и предбедро), сзади; 29 — правая вульва, сзади; 30 - то же, дистально; 31 - то же, частично вместе с левой, вид спереди и дистально. Масштаб 0,1 мм. 
Quant aux bourses, elles se présentent différemment sur les deux vulves, non pour des raisons structurelles, mais sans doute physiologiques: celle de gauche est fermée, les trois pièces, l'opercule et les deux valves de la bourse étant serrées les unes contre les autres. Sur la vulve droite, au contraire, les trois pièces sont écartées l'une de l'autre, de telle sorte que l'on peut d'une part, observer le large espace concédé par l'écartement des deux valves (ve et vi) entre elles, et d'autre part, voir sur l'extrémité orale de chaque valve, un téton (z) qui suggère la paire de boutons-pression de fermeture observés (rarement) chez certains chordeumatides.

Affinités: Manifestement distincte de la précédente par plusieurs détails de structures gonopodiales et paragonopodiales, cette espèce en est cependant très proche (et pas seulement du point de vue géographique). De ce fait, elle se rattache aussi au groupe des quatre béticorifaines, dont elle possède, entre autres caractères, les télopodites gonopodiaux bifides et entrecroisés caractéristiques de ce groupe. De ce fait, nos deux espèces nouvelles se distinguent de leurs proches voisines géographiques, telle que $C$. solanasi, chez qui les télopodites gonopodiaux ne sont ni arqués, ni bifides, ni entrecroisés, ou encore de $C$. jabalensis, chez qui les télopodites gonopodiaux sont bifides et remarquables par la réduction à de simples digitations érigées de la branche principale.

Clé des espèces du genre Ceratosphys, mise à jour La clé publiée dans Mauriès [2013a: 55] est modifiée comme suit du point 10 au point 15 , pour y introduire C. jabaliensis Mauriès, 2013 et les deux espèces nouvelles. Les cavernicoles sont indiqués par un $*$, les nivicoles par **.

10. Préfémur des P.9 (paragonopodes) simple .............. 11 - Préfémur des P.9 orné d'une expansion ventrale parfois importante ........................................................... 14 $14 \mathrm{c}$

11. Aux P.8 (gonopodes): bord distal du bouclier syncolpocoxal largement et profondément échancré en $\mathrm{V}$..... ...................... C. solanasi Mauriès \& Vicente, 1977*

Espagne, prov. Albacete, Riopar, grottes.

- Aux P.8: bord distal du bouclier syncolpocoxal non ou faiblement échancré ............................................. 12

12. Aux P.8: synangiocoxite en bandeau ou ceinture basale caudale ornée de deux lobes de chaque côté C. banyulensis Brölemann, 1926

France, dpt. Pyrénées-Orientales, Banyuls-sur-Mer.

- Aux P.8: synangiocoxite constitué de deux masses parasagittales plus ou moins largement soudées médialement

13

13. Aux P.8: télopodites gonopodiaux nettement divisés en deux rameaux qui s'entrecroisent .............................. 14

- Aux P.8: télopodites non bifides

C. soutadei Mauriès, $1970 * *$

Espagne, prov. Granada, Sierra Nevada

14. Aux P.9 (paragonopodes): préfémur relativement grêle, presque 4 fois plus long que large. Aux P.8: bord distal du bouclier syncolpocoxal en deux lobes parasagittaux séparés par une minuscule échancrure médiane

C. deharvengi Mauriès, 1978**

Espagne, prov. Màlaga, Sierra de las Nieves.
- Aux P.9: préfémur environ deux fois plus long que large. Aux P.8: bord distal du bouclier syncolpocoxal faiblement échancré ................................................. 14a

14a. Aux P.9: préfémur claviforme ...... C. fernandoi sp.n.* Espagne, prov. Granada, Castril, grotte.

- Aux P.9: préfémur non claviforme, plus large à sa base qu'à l'apex ......................................................... 14b

14b. Aux P.9: préfémur presque deux fois plus long que le podomère distal. Aux P.8: les deux moitiés du synangiocoxite sont séparées distalement par une échancrure en U ................................... C. nodipes (Attems, 1952)**

Espagne, prov. Málaga, Sierra Ronda.

- Aux P.9: préfémur à peine plus long que le podomère distal. Aux P.8: les deux moitiés du synangiocoxite sont séparées distalement par un petit lobe médian C. flammeola sp.n.*

Espagne, prov. Jaén, Cazorla, grotte.

14c. Aux P.9: préfémur pourvu d'un appendice ventral presque aussi long que lui, en simple tige émoussée apicalement C. jabaliensis Mauriès, 2013*

Espagne, prov. Jaén, Pontones, grotte. - Aux P.9: appendice ventral du préfémur d'une autre forme: en lobe subtriangulaire, ou subquadrangulaire, en grand lobe arrondi ou encore plus complexe ..................... 15

15. Rameau ventral du préfémur...etc..

\section{Conclusion}

La Figure 32 ne donne qu'une idée très partielle et imparfaite de la répartition des Diplopodes en Andalousie; car elle ne prétend pas cartographier tous les diplopodes répertoriés dans cette région, mais seulement à représenter la répartition des quatre genres qui y ont fourni récemment par Mauriès [2013b], par Golovatch \& Mauriès [2013] et par la présente note, des taxons nouveaux. On notera aussi qu'avant ces trois notes, où ne figurent ni les Glomerida ni les Callipodida, la soixantaine d'espèces de diplopodes répertoriées en Andalousie, l'a été surtout sur les trois provinces du sud, Càdiz, Granada et Màlaga. La province de Jaén est la grande bénéficiaire de l'apport effectué par le GEV, qui a surtout prospecté au Nord-ouest de la province, dans et autour du Parc naturel de Cazorla, Segura y Las Villas.

On notera l'apport quantitatif et qualitatif constitué par les espèces du genre Ceratosphys, dont on connaît le fort taux d'endémisme et qui, en Andalousie, est représenté par des espèces montagnardes, voire nivicoles au sud-est de la province, et par des cavernicoles dans le nord-ouest. Ces niches-refuges illustrent bien les conclusions de Mauriès [2013a], qui considère le genre, avec sa grande aire discontinue de la Belgique au Maroc, comme chorologiquement en régression.

L'intérêt des trois autres genres n'est pas moindre, même s'il paraît à première vue plus limité. Ainsi le genre Archipolydesmus passe de une à 4 espèces distinctes (dont une non nommée) pour l'Andalousie, Origmatogona passe de une à deux, et Psichrosoma est nouveau pour la province.

Pour faire un bilan des récoltes effectuées en Andalousie par le GEV, on se reportera, en complément 


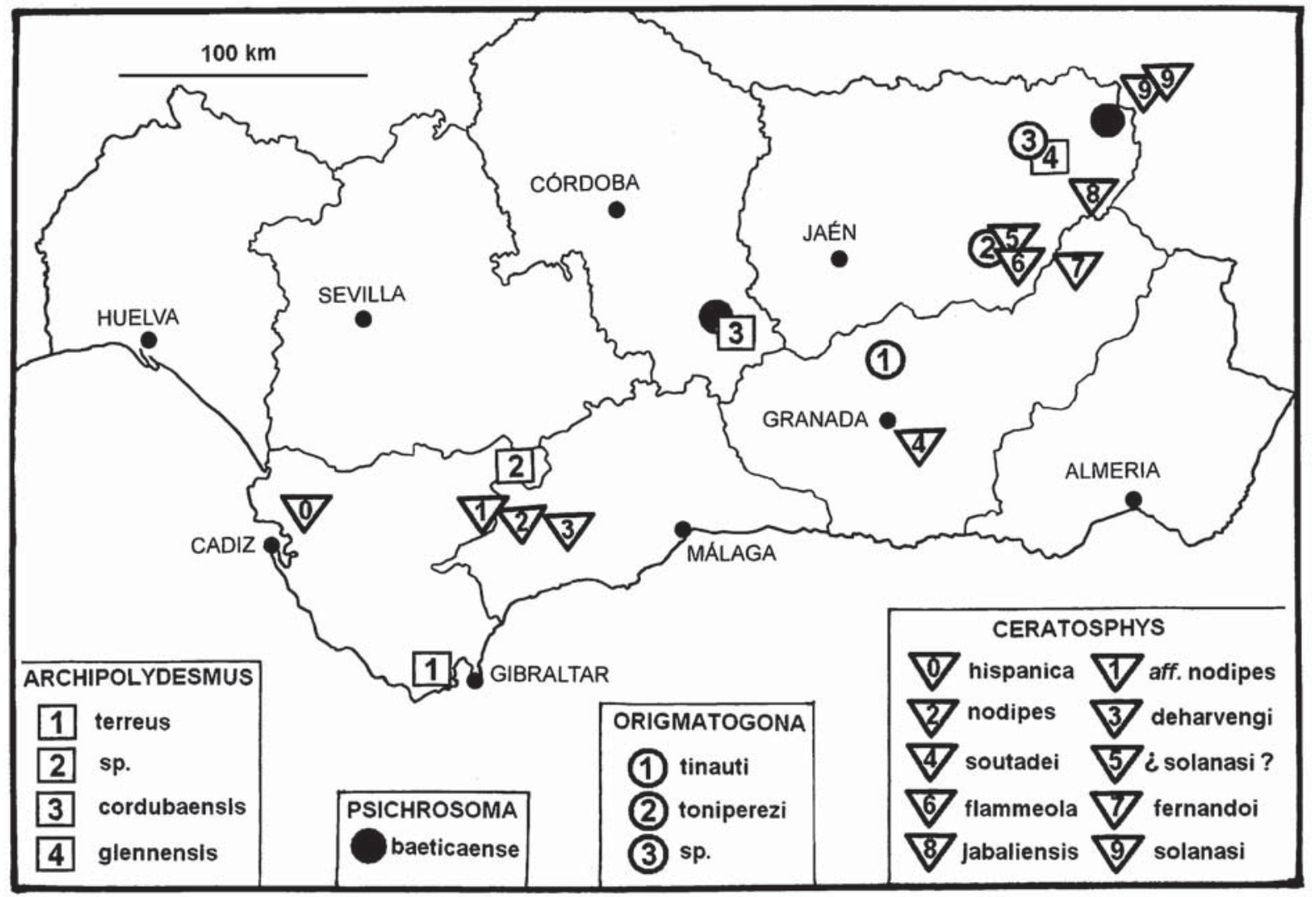

Fig. 32. Carte de répartition en Andalousie des 4 genres (Archipolydesmus, Origmatogona, Psichrosoma et Ceratosphys) qui ont fourni récemment des taxa nouveaux dans cette région. A noter que les deux localités de $C$. solanasi (triangles ${ }^{\circ} 9$ ) se trouvent légèrement à l'extérieur de l'Andalousie.

Fig. 32. Map showing the distribution in Andalusia of 4 genera (Archipolydesmus, Origmatogona, Psichrosoma and Ceratosphys) which have recently gained in new taxa in that region. It is noteworthy that both localities of $C$. solanasi (triangles No. 9) lie slightly beyond Andalusia.

Рис. 32. Карта с распространением в Андалусии четырех родов (Archipolydesmus, Origmatogona, Psichrosoma и Ceratosphys), которые в этом регионе недавно пополнились новыми таксонами. Стоит отметить, что обе точки находок C. solanasi (треугольники № 9) лежат слегка за пределами Андалусии.

de la présente note, à Golovatch \& Mauriès [2013] et à Mauriès [2013b]. Ces progrès récents très prometteurs laissent présager que de futures prospections seront encore très fructueuses, non seulement dans les provinces les mieux connues mais plus encore et surtout dans les autres. Elles contribueront ainsi à contribuer efficacement aux initiatives nationales (cf. Projet Fauna Ibérica) et européennes [cf. Kime \& Enghoff, 2011].

REMERCIEMENTS. Il m'est agréable de rendre hommage ici au Grupo de Espeleología de Villacarillo (G.E.V.), et à son animateur, Antonio Perez Fernandez, dont les activités ont permis d'accroître sensiblement nos connaissances sur la faune diplopodologique de l'Andalousie et notamment sur les cavernicoles qui constituent une part importante de ces découvertes. Ces spéléologues ont été aidés par une subvention accordée par l'excellentissime Députation de la province de Jaén et par l'institut de "Estudios Giennense". Ils ont été soutenus et encouragés par le Conseil de l'Environnement de l'Assemblée d'Andalousie et par celui du Parc Naturel des Sierras de Cazorla, Segura et Las Villas.
L'auteur tient à remercier aussi son collègue et ami Serge Golovatch, qui a bien voulu lui confier l'étude de ces matériaux, dont la plus grande partie va enrichir les collections du Musée Zoologique de Moscou.

\section{Littérature}

Abrous-Kherbouche O., Mauriès J.-P. 1996. Découverte du genre Archipolydesmus en Algérie: description de trois espèces nouvelles (Diplopoda, Polydesmida, Polydesmidae) // Bulletin du Muséum national d'Histoire naturelle, Paris, $4^{\text {ème }}$ série. T.18A. Nos 3-4. P.571-587.

Attems C. 1898. System der Polydesmiden. I. Theil // Denkschriften der Mathematisch-Naturwissenschaftlichen Classe der Kaiserlichen Akademie der Wissenschaften, Wien. Bd.67. S.221-482.

Attems C. 1952. Myriopoden der Forschungsreise Dr. H. Franz in Spanien 1951 nebst Übersicht über die gesamte iberische Myriopodenfauna // Eos, Madrid. T.28. No.4. P.323-366.

Bollman C.H. 1888. Notes upon a collection of Myriapoda from East Tennesee, with description of a new genus and six new species // Annals of the New York Academy of Sciences. Vol.10. P. 106-112.

Brölemann H.W. 1900. Myriapodes recueillis en Espagne par le P.J. Pantel // Bulletin de la Société Entomologique de France. No.6. P.131-133. 
Brölemann H.W. 1902. Description d'un nouveau Polydesme d'Espagne // Bulletin de la Société Entomologique de France. No.1. P.13-14.

Brolemann H. W. 1926. Myriapodes des Pyrénées-Orientales // Bulletin de la Société d'Histoire Naturelle de Toulouse. T.54. No.2. P.233-267.

Ceuca T. 1968. Archipolydesmus osellai (Diplopoda, Polydesmidae). Une nouvelle espèce montagnarde de l'Espagne // Memorie del Museu Civico di Storia Naturale, Verona. T.16. P.135-140.

Ceuca T. 1988. Sur quelques diplopodes de la péninsule Ibérique et du nord de l'Afrique // Studia Universitațea Babeş-Bolyai, Biol. T.33. No.2. P.40-48.

Golovatch S.I., Mauriès J.-P. 2013. Los diplópodos (Myriapoda, Diplopoda) subterràneos de Jaén // Los invertebratos subterràneos de Jaén, Grupo de Espeleología de Villacarillo. P.88-93.

Kime D.R., Enghoff H. 2011. Atlas of European Millipedes (Class Diplopoda), Volume 1 // Fauna Europaea Evertebrata, No.3. Pensoft: Sofia-Moscow, P.1-282.

Manfredi P. 1956. Miriapodi cavernicoli del Marocco, della Sardegna, e del Piemonte // Atti della Società Italiana di Scienze Naturali, Milano. T.95. Nos 3-4. P.197-222.

Mauriès J.-P. 1964. Sur quelques Diplopodes de la péninsule Ibérique // Bulletin de la Société d'Histoire Naturelle de Toulouse. T.99. Nos 1-2. P.157-170.

Mauriès J.-P. 1966. Vandeleuma et Vascosoma, genres nouveaux des grottes du Pays Basque français (Diplopoda-Craspedosomoidea) // Annales de Spéléologie. T.21. No.3. P.631-641.

Mauriès J.-P. 1968. Documents inédits du Professeur H. Ribaut. I. Description de Ceratosphys hispanica Ribaut, 1920 (Diplopoda, Craspedosomoidea) // Bulletin de la Société d'Histoire Naturelle de Toulouse. T.104. Nos 1-2. P.65-68.

Mauriès J.-P. 1970. Myriapodes de Sierra Nevada (Espagne). Une nouvelle espèce du genre Ceratosphys Ribaut, 1920 (Diplopoda) // Publicaciones del Instituto de Biologia Aplicada, Barcelona. T.47. P.131-139.

Mauriès J.-P. 1978. Myriapodes Diplopodes du sud de l'Espagne. Description d'une espèce nouvelle, d'espèces mal connues et révision de types du Museum de Vienne // Annalen des Naturhistorischen Museums in Wien. Bd.81. S.575-588.

Mauriès J.-P. 1981. Diplopodes épigés et cavernicoles des Pyrénées espagnoles et des Monts cantabriques. IX. Asturasoma, nou- veau genre (Diplopoda, Craspedosomida) // Bulletin de la Société d'Histoire Naturelle de Toulouse. T.117. Nos 1-4. P.162170.

Mauriès J.-P. 1985. Polydesmide et Craspedosomides cavernicoles nouveaux de France et du Maroc (Myriapoda Diplopoda) // International Journal of Speleology. T.14. Nos 1-4. P.51-62 (for 1984-1985)

Mauriès J.-P. 1990a. Diplopodes de la péninsule Ibérique: deux espèces nouvelles du genre Ceratosphys Ribaut, 1920 (Diplopoda, Craspedosomida, Opisthocheiridae) // Miscellanea Zoologica, Barcelona. T.14. P.115-123.

Mauriès J.-P. 1990b. Révision des Origmatogonini. Taxons nouveaux de France et d'Espagne (Diplopoda, Craspedosomida, Chamaesomidae // Bulletin de la Société d'Histoire Naturelle de Toulouse. T.126. P.47-60.

Mauriès J.-P. 2013a. Le genre Ceratosphys Ribaut, 1920: trois nouveaux taxa de Catalogne et des îles Baléares (Diplopoda, Craspedosomatida, Opisthocheiridae) // Bulletin de la Société d'Histoire Naturelle de Toulouse. T.148. P.47-57.

Mauriès J.-P. 2013b. Trois espèces nouvelles de Diplopodes cavernicoles de 1'Andalousie (Espagne) (Diplopoda: Polydesmida: Polydesmidae; Chordeumatida: Vandeleumatidae, Opisthocheiridae) // Arthropoda Selecta. T.22. No.2. P.97-112.

Mauriès J.-P., Geoffroy J.-J. 1982. Découverte, dans les Causses Majeurs, d'une remarquable espèce du genre Opisthocheiron Ribaut, 1913 // Bulletin de la Société d'Histoire Naturelle de Toulouse. T.118. P.131-140.

Mauriès J.-P., Vicente M.-C. 1977. Diplópodos cavernicolos nuevos y poco conocidos de España, recolectados por A. Lagar. Descripción de très géneros nuevos // Miscellanea Zoologica, Barcelona. T.4. No.1. P.109-134.

Ribaut H. 1913. Biospeologica ${ }^{\circ}$ XXVIII: Ascospermophora (Myriapodes) (Première série) // Archives de Zoologie expérimentale et générale, Paris. T.10. No.8. P.399-478, pl.31-47.

Ribaut H. 1920. Notes sur les Chordeumoïdes de France // Bulletin de la Société d'Histoire Naturelle de Toulouse. T.48. No.1 P.18-43.

Vicente M.C. 1981. Diplópodos epigeos de Cataluña (Glomeridos, Craspedosómidos y Polidésmidos) // Eos. T.57. P.279-315.

Responsible editor S.I. Golovatch 
Annexe. Identifications des Diplopodes récoltés par le G.E.V. de 2008 à 2013. Appendix. Identifications of Diplopoda collected by the G.E.V. between 2008 and 2013. Приложение. Определения Diplopoda, собранных G.E.V. между 2008 и 2013 гг.

\begin{tabular}{|c|c|c|c|c|}
\hline \multicolumn{3}{|r|}{ PROVINCES, Localités, sites } & dates & det. JPM 2013 \\
\hline \multirow{14}{*}{$\begin{array}{l}\text { A } \\
\text { L } \\
\text { B } \\
\text { A } \\
\text { C } \\
\text { E } \\
\text { T } \\
\text { E }\end{array}$} & \multirow{7}{*}{\begin{tabular}{|l|} 
Cueva \\
de \\
los \\
$\mathrm{C}$ \\
$\mathrm{H}$ \\
$\mathrm{O}$ \\
$\mathrm{R}$ \\
$\mathrm{R}$ \\
$\mathrm{O}$ \\
$\mathrm{S}\left({ }^{*}\right)$ \\
\end{tabular}} & Paso del 16 Agosto & $\begin{array}{c}80810 \\
/ 50910\end{array}$ & Proceratosphys solanasi $10 \sigma^{7} \sigma^{7}, 12$ $+9,7 \mathrm{jj}$. \\
\hline & & Paso del 16 Agosto & $\begin{array}{l}40710 \\
/ 80810\end{array}$ & idem. $1 \sigma^{7}$ préadulte, $16 \sigma^{7} \sigma^{7}, 15$ Oᄋ, 15 jj. \\
\hline & & Cabrera, Paso del 16 Agosto & \multirow{3}{*}{$\begin{array}{c}80810 \\
/ 50910\end{array}$} & idem. 19 \\
\hline & & Zona Lago largo / Lago verde & & idem. $10^{7}$ \\
\hline & & - & & idem. dépigmenté 4 O+, 1 j.(stade VI) \\
\hline & & Sala de la Colada & \multirow{2}{*}{$\begin{array}{l}40710 \\
/ 80810\end{array}$} & idem 1 + pigmentée \\
\hline & & Sala del Comprimento & & idem 1 q. dépigmentée \\
\hline & \multirow{7}{*}{\begin{tabular}{|l|} 
Cueva \\
del \\
F \\
A \\
R \\
A \\
LL \\
O \\
N
\end{tabular}} & Sala Alcoy & \multirow{7}{*}{$\begin{array}{l}250510 \\
/ 220810\end{array}$} & idem $13 \bigcirc^{7} \sigma^{7}, 8$ 우, 15 jj. \\
\hline & & Sala Crevillante & & idem $1 \mathrm{O}^{7}$ bien pigmenté \\
\hline & & Via latéral ... entrada & & idem $1 \bigcirc^{7}, 1$ +, $1 \mathrm{j} .($ stadeVII) \\
\hline & & Sala de las Espadas & & idem $1 \bigcirc^{r}$ \\
\hline & & Zona proxima del entrada & & idem 1 +, $10^{7} \& 1$ \$ préad., 1 j. (stade VII) \\
\hline & & Salto del gato & & idem. $1 \bigcirc^{2}, 1+, 1 \sigma^{2} \& 19$ préadultes, $5 \mathrm{jj}$. \\
\hline & & - & & idem $1 \sigma^{7}, 1$ +, 2 jj. dépigmentés \\
\hline \multicolumn{3}{|c|}{$\begin{array}{l}\text { CADIZ, Villaluenga del Rosario, } \\
\text { Sistema Republicano-Cabito }\end{array}$} & 230411 & $\begin{array}{l}\text { Ceratosphys aff. nodipes Attems } 1 O^{7}, 1 O^{7} \mathrm{j} ., 3 \\
\text { of } 19 \mathrm{j} .\end{array}$ \\
\hline \multicolumn{3}{|c|}{$\begin{array}{l}\text { GRANADA, Castril } \\
\text { Cueva de Don Fernando }\end{array}$} & 20509 & $\begin{array}{l}\text { Ceratosphys fernandoi sp.n. } 4 \bigcirc^{7} \sigma^{7}, 1 \sigma^{7} \text { j., } 1 \text {, } \\
4 \text { j.(stade VII) holo-, allo- \& paratypes }\end{array}$ \\
\hline \multirow{6}{*}{\multicolumn{2}{|c|}{ JAEN }} & Hornos, Sima de los Moluscos & 280412 & Origmatogona (?) sp. une moitié post. \\
\hline & & \multirow[t]{3}{*}{$\begin{array}{l}\text { Cazorla, Cueva secreta del Poyo } \\
\text { Manquillo }\end{array}$} & $\begin{array}{c}\text { Muestreo } \\
\text { Manval } \\
311010 \\
\end{array}$ & $\begin{array}{l}\text { Origmatogona toniperezi } \text { sp.n. } 1 \sigma^{7}, 1+, 1 \% \\
\text { préadulte holo-, allo- \& paratypes }\end{array}$ \\
\hline & & & 101010 & $\begin{array}{l}\text { Origmatogona toniperezi } \mathbf{s p . n . ~} 2+9,19 \mathrm{j} \\
\text { topotypes } \\
\text { Ceratosphys sp.(solanasi } ?) 1+ \\
\end{array}$ \\
\hline & & & 120611 & $\begin{array}{l}\text { Origmatogona toniperezi sp.n. } 4 \bigcirc^{7} \sigma^{7}, 5 \text { } 50,1 \\
\text { j. topotypes }\end{array}$ \\
\hline & & $\begin{array}{l}\text { Péal de Becerro, Complejo del Arroyo de } \\
\text { la Rambla (PB-4) }\end{array}$ & 41211 & $\begin{array}{l}\text { Psichrosoma sp. (baeticaense ?) } 1 \text { juv. (stade } \\
\text { VII) }\end{array}$ \\
\hline & & Siles, Cueva del Nacimiento de San Blas & 190709 & $\begin{array}{l}\text { Psichrosoma baeticaense1 juv. préad., } 1 \text { juv. } \\
\text { (stade VII) }\end{array}$ \\
\hline \multirow{3}{*}{\multicolumn{2}{|c|}{ JAEN }} & Hornos, Sima del Campamento & 30313 & Archipolydesmus giennensis sp.n. $10^{7}$ holotype \\
\hline & & Cazorla, Cueva Moreno & 140413 & Ceratosphys flammeola sp.n. $1 \bigcirc^{7}$ holotype \\
\hline & & $\begin{array}{l}\text { Cazorla, Cueva secreta del Poyo } \\
\text { Manquillo }\end{array}$ & 140413 & Origmatogona toniperezi sp.n. 2 juv. topotypes \\
\hline \multirow{2}{*}{\multicolumn{2}{|c|}{ CADIZ }} & $\begin{array}{l}\text { Villaluenga del Rosario, } \\
\text { Sistema Republicano-Cabito }\end{array}$ & 230411 & Blaniulidae $1+1 \mathrm{j}$., fragments \\
\hline & & Villaluenga d. Rosario, Sima Cacao & 210411 & Acipes sp. $1 \bigcirc^{1}$ j. 4 O+, 1 j., fragments \\
\hline \multicolumn{3}{|c|}{ MALAGA, Antequera, Cueva del Yeso III } & 270408 & Dolichoiulus sp. 19 \\
\hline
\end{tabular}

(*) loc. type de Proceratosphys solanasi Mauriès et Vicente, 1977 